\title{
The directional instability problem in systems with frictional contacts
}

\author{
A. Pinto da Costa ${ }^{a, *}$, J.A.C. Martins ${ }^{\text {a }}$, I.N. Figueiredo ${ }^{b}$, J.J. Júdice ${ }^{b}$ \\ a Dep. Eng. Civil e Arquitectura and I.C.I.S.T., Instituto Superior Técnico, Av. Rovisco Pais, 1049-001 Lisboa, Portugal \\ b Dep. de Matemática, Universidade de Coimbra, Apartado 3008, 3001-454 Coimbra, Portugal
}

Received 16 June 2003; accepted 29 September 2003

\begin{abstract}
The research summarized in this paper addresses the directional instability of finite dimensional systems with unilateral frictional contacts. Conditions for the occurrence of this divergence type instability are discussed, complementarity formulations are developed, and numerical procedures are proposed for the solution of the corresponding non-smooth stability eigenproblems. Various examples are analytically or numerically solved and discussed, namely some finite element examples that have instability modes involving evolution towards slip or stick in different portions of the contact surface.
\end{abstract}

(c) 2003 Elsevier B.V. All rights reserved.

Keywords: Coulomb friction; Directional instability; Angular bifurcation; Complementarity problems; Finite elements

\section{Introduction}

In continuation of the previous works $[19,20]$, the present paper addresses the directional instability of equilibrium states of finite-dimensional non-linear elastic systems with unilateral frictional contacts.

The study of instabilities and bifurcations in systems with friction has been motivated by many experimental observations related to technological problems or industrial processes, like the squeal of brakes $[10,11]$ or rubber/glass contacts [39] and the intermittence of granular flows [9]. Experimental observations of stress waves [32,40] in a rectangular polyurethane block pressed against an araldite surface motivated the theoretical and numerical studies in [19,31]. The practical importance of the instability phenomena in frictional contact systems has led to numerous studies, referenced, for instance, in [6,13, 14]. In the last decades the scientific community has also become progressively aware of the fact that friction-induced instability phenomena are essential ingredients of the source mechanism of earthquakes [4,34-36].

\footnotetext{
${ }^{*}$ Corresponding author.

E-mail addresses: apcosta@civil.ist.utl.pt (A. Pinto da Costa),jmartins@civil.ist.utl.pt (J.A.C. Martins), isabelf@mat.uc.pt (I.N. Figueiredo), joaquim.judice@co.it.pt (J.J. Júdice).
} 
In what concerns divergence type instabilities with classical unilateral contact and Coulomb's friction law, the first theoretical results were due to Chateau and Nguyen [5], who established a sufficient condition for the absence of this type of instability for a continuous elastic body. An important study on the stability of finite dimensional non-linear elastic systems with unilateral contact but without friction was due to Klarbring [15]. Klarbring [15,18] and Björkman [3] studied the occurrence of bifurcations and several critical points in quasi-static trajectories of discrete systems with frictionless contacts. Mróz and Plaut [23] studied the stability of elastic structures with friction assuming known normal reactions, which eliminated the non-associative character of the general problems of contact with friction. Some interesting finite dimensional examples that do not have that limitation are presented in [24]. The study of flutter instabilities in frictional contact systems is outside the scope of the present paper and the corresponding references will not be mentioned here.

The similarities between the mechanics of structures with contact and friction and the mechanics of elastic-plastic structures furnishes an additional motivation for the study of the stability of frictional systems. Recent surveys on bifurcation and stability of dissipative solids can be found in [28,30]. Some aspects of the cross fertilization between that field and the bifurcation and stability of frictional contact systems are cited in [21].

The main contributions of the present paper to the study of directional instabilities in finite dimensional frictional contact problems are the following:

- their formulation as non-smooth (complementarity) eigenproblems;

- the transformation of these into mixed complementarity problems ( $M C P s)$, and the numerical resolution of the latter with the PATH Mathematical Programming algorithm;

- the finding and preliminary discussion of modes of directional instability in finite element systems containing portions of the contact surface that evolve towards sliding and others towards stick;

- the establishment of a relation between bifurcation of quasi-static trajectories and directional instability in frictional contact systems that takes into account the intrinsic non-symmetry of these problems and thus improves on earlier results.

The organization of the rest of this paper is as follows. The next section deals with the kinematics of finite dimensional plane mechanical systems in the presence of rigid curved fixed obstacles. Section 3 is devoted to the governing equations for smooth dynamic motions and static equilibria, and Section 4 to the relevant sets of the admissible kinematic and static variables. The study of the directional instability of equilibrium states involves the analysis of the existence of dynamic solutions with initial conditions arbitrarily close to such equilibrium states and diverging from them in a non-oscillatory manner. In Section 5, this kind of instability is studied for non-linear elastic systems by analysing the directionally linearized system in the neighborhood of such static equilibrium states. This leads to the formulation and resolution of non-smooth eigenproblems. Section 6 is devoted to their formulation as complementarity eigenproblems, followed by their transformation into mixed complementarity problems $(M C P S)$ and their numerical solution with the PATH Mathematical Programming algorithm. Section 7 presents instability modes for finite element models of, namely, a tribology experiment and the well known single particle example of Klarbring. Section 8 relates the directional instability problem and the quasi-static first order rate problem, by using their complementarity formulations.

\section{Kinematics}

In this study we consider a plane holonomic finite dimensional non-linear elastic system. At each time $t$ its configuration is described by the vector $\mathbf{X}(t) \in \mathbb{R}^{N}$ of generalized coordinates, which are independent in 


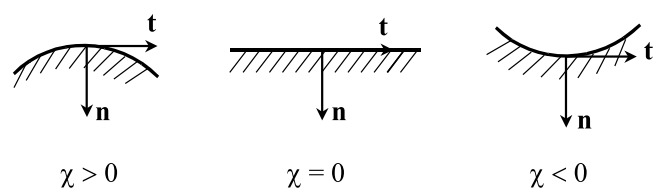

Fig. 1. Sign convention for the obstacle curvature $\chi$.

the absence of unilateral constraints. A finite number of particles of the system may have unilateral Contact with smooth curved fixed obstacles: the labels $p$ of such particles are grouped in the set $\mathscr{P}_{C} \subset \mathbb{N}$ $\left(\# \mathscr{P}_{C}=n_{C}\right)$. For each particle $p$ candidate to contact, the corresponding obstacle is identified by the set of vectors $\mathbf{X} \in \mathbb{R}^{N}$ such that $\Phi^{p}(\mathbf{X})=0$, where $\Phi^{p}$ is a smooth scalar function, $\mathbf{n}^{p}$ and $\mathbf{t}^{p}$ denote the unit vectors that are normal and tangential to the obstacle, respectively; the vector $\mathbf{n}^{p}$ points towards the interior of the obstacle. The curvature of the obstacle $p$ is denoted by $\chi^{p}$, whose sign is given in Fig. 1. The components of the velocity and reaction vectors of all contact candidate particles in the local bases $\left(\mathbf{n}^{p}, \mathbf{t}^{p}\right)$ are grouped in the vectors $\mathbf{v}$ and $\mathbf{r}$, which belong to $\mathbb{R}^{2 n_{C}}$. The relations between the vectors $\mathbf{v}$ and $\mathbf{r}$ and their generalized counterparts $\mathbf{V}$ and $\mathbf{R}$ are expressed by $\mathbf{v}=\mathbf{G V}$ and $\mathbf{R}=\mathbf{G}^{\mathrm{T}} \mathbf{r}$ [20]. It is assumed that

the lines of the $2 n_{C} \times N$ matrix $\mathbf{G}$ are linearly independent.

Assumption (1) guarantees that: (i) any contact velocity vector $\mathbf{v}$ can be obtained from a vector of generalized velocities by means of the kinematic transformation $\mathbf{v}=\mathbf{G V}$; and (ii) a vanishing vector of generalized reactions $\mathbf{R}$ implies a vanishing vector $\mathbf{r}$ of reactions at the contact candidate particles. In these circumstances the linear map $\mathbf{G}(\mathbf{X}): \mathbb{R}^{N} \rightarrow \mathbb{R}^{2 n_{C}}$ is subjective, has a right inverse, and it is possible to perform a change of variables [20]

$$
\mathbf{V}=\mathbf{T} \widetilde{\mathbf{V}},
$$

where the matrix $\mathbf{T} \in \mathbb{R}^{N \times N}$ [20] is non-singular, the transformed vector of generalized velocities $\tilde{\mathbf{V}}$ has the decomposition $\widetilde{\mathbf{V}}=\left[\begin{array}{ll}\mathbf{V}_{F}^{\mathrm{T}} & \mathbf{v}^{\mathrm{T}}\end{array}\right]^{\mathrm{T}}$, the $n_{F}$ generalized velocities in $\mathbf{V}_{F}\left(n_{F}=N-2 n_{C}\right)$ are Free from any kinematic unilateral constraint, and $\mathbf{v}$ groups the normal and tangential velocities of the contact candidate particles. Similarly, the transformed vector of generalized reactions $\widetilde{\mathbf{R}}$ has the decomposition $\widetilde{\mathbf{R}}=\left[\begin{array}{ll}\mathbf{0}^{\mathrm{T}} & \mathbf{r}^{\mathrm{T}}\end{array}\right]^{\mathrm{T}}$, where $\mathbf{0}$ is the null vector of dimension $n_{F}$. Note that when the displacements normal and tangential to (flat) obstacles of all the contact candidate particles are taken as generalized coordinates (as in some finite element discretizations), the matrix $\mathbf{G}$ is a boolean matrix.

\section{Governing dynamic and static equations}

For any contact candidate particle $p \in \mathscr{P}_{C}$, the classical unilateral contact conditions and the friction law of Coulomb at some time $t \geqslant 0$ are

$$
\Phi^{p}(\mathbf{X}(t)) \leqslant 0, \quad r_{n}^{p}(t) \leqslant 0, \quad \Phi^{p}(\mathbf{X}(t)) r_{n}^{p}(t)=0
$$

and

$$
r_{t}^{p}(t) \in \mu r_{n}^{p}(t) \sigma\left[v_{t}^{p}(t)\right],
$$

respectively; $\mu \geqslant 0$ is the coefficient of friction, $\sigma[\cdot]$ denotes the multi-valued application such that, for each $x \in \mathbb{R}$,

$$
\sigma[x]= \begin{cases}x /|x|, & \text { if } x \neq 0 \\ {[-1,+1],} & \text { if } x=0\end{cases}
$$


The friction law can be alternatively written in the equivalent form of an inequality plus an equality:

$$
\left\{\begin{array}{l}
\left|r_{t}^{p}(t)\right|+\mu r_{n}^{p}(t) \leqslant 0 \\
r_{t}^{p}(t) v_{t}^{p}(t)-\mu r_{n}^{p}(t)\left|v_{t}^{p}(t)\right|=0
\end{array}\right.
$$

During the smooth portions of its dynamic evolution the system is governed by the Lagrange equations

$$
\mathbf{M}(\mathbf{X}(t)) \ddot{\mathbf{X}}(t)=\mathbf{F}(\mathbf{X}(t), \dot{\mathbf{X}}(t))+\mathbf{G}^{\mathrm{T}}(\mathbf{X}(t)) \mathbf{r}(t),
$$

where $t \geqslant 0$ is the time variable, $\mathbf{M}$ is the $N \times N$ symmetric positive definite $(P D)$ mass matrix, $\mathbf{F}$ is the vector of constant external applied forces, internal elastic forces, and, in general mechanical systems, quadratic terms involving the generalized velocities, ( $)$ denotes the time derivative and $\mathbf{X}(t)$ and $\mathbf{r}(t)$ are the vectors of the unknown generalized coordinates and contact reactions at time $t$. During smooth dynamic evolutions, Eqs. (7) are satisfied together with the laws (3) and (4) (or (6)) and with the appropriate initial conditions on the configuration and on the velocity. An equilibrium state of the system is characterized by a configuration vector $\mathbf{X}^{0}$ and a reaction vector $\mathbf{r}^{0}$ that satisfy (7), (3) and (4) with $\dot{\mathbf{X}}(t)=\ddot{\mathbf{X}}(t) \equiv 0$.

\section{Some relevant sets of admissible variables}

The set of admissible configurations is defined by

$$
\mathscr{K}_{\mathbf{X}} \doteq\left\{\mathbf{X} \in \mathbb{R}^{N}: \Phi^{p}(\mathbf{X}) \leqslant 0 \text { for all } p \in \mathscr{P}_{C}\right\} .
$$

For each $\mathbf{X} \in \mathbb{R}^{N}$ and each $\mathbf{r} \in \mathscr{K}_{\mathbf{r}}(\mathbf{X})$ the decomposition of the set $\mathscr{P}_{C}$ of contact candidate particles is introduced

$$
\begin{aligned}
& \mathscr{P}_{C}=\mathscr{P}_{f}(\mathbf{X}) \cup \mathscr{P}_{z}(\mathbf{X}, \mathbf{r}) \cup \mathscr{P}_{d}(\mathbf{X}, \mathbf{r}) \cup \mathscr{P}_{s}(\mathbf{X}, \mathbf{r}), \\
& \mathscr{P}_{f}(\mathbf{X}) \doteq\left\{p \in \mathscr{P}_{C}: \Phi^{p}(\mathbf{X})<0\right\} \quad\left[\text { particles currently not in contact }(\text { free }), \# \mathscr{P}_{f}=n_{f}\right], \\
& \mathscr{P}_{z}(\mathbf{X}, \mathbf{r}) \doteq\left\{p \in \mathscr{P}_{C}: \Phi^{p}(\mathbf{X}) \geqslant 0, r_{n}^{p}=r_{t}^{p}=0\right\}
\end{aligned}
$$

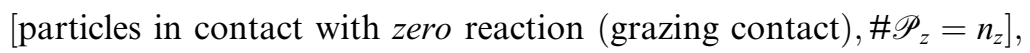

$$
\begin{aligned}
& \mathscr{P}_{d}(\mathbf{X}, \mathbf{r}) \doteq\left\{p \in \mathscr{P}_{C}: \Phi^{p}(\mathbf{X}) \geqslant 0, r_{n}^{p}<0 \text { and }\left|r_{t}^{p}\right|<-\mu r_{n}^{p}\right\} \\
& \mathscr{P}_{s}(\mathbf{X}, \mathbf{r}) \doteq\left\{p \in \mathscr{P}_{C}: \Phi^{p}(\mathbf{X}) \geqslant 0, r_{n}^{p}<0 \text { and }\left|r_{t}^{p}\right|=-\mu r_{n}^{p}\right\}
\end{aligned}
$$

In what concerns the reaction forces, we define, for each $\mathbf{X} \in \mathbb{R}^{N}$, the (configuration dependent) set of admissible reaction forces:

$$
\begin{aligned}
\mathscr{K}_{\mathbf{r}}(\mathbf{X}) \doteq\left\{\mathbf{r} \in \mathbb{R}^{2 n_{C}}: r_{n}^{p}=r_{t}^{p}=0 \quad \text { for all } p \in \mathscr{P}_{f}(\mathbf{X}) ; \quad r_{n}^{p} \leqslant 0 \text { and }\left|r_{t}^{p}\right|+\mu r_{n}^{p} \leqslant 0 \quad \text { for all } p \in \mathscr{P}_{C}\right. \\
\text { such that } \left.\Phi^{p}(\mathbf{X}) \geqslant 0\right\} .
\end{aligned}
$$

For $\mathbf{X} \in \mathbb{R}^{N}$ and $\mathbf{r} \in \mathscr{K}_{\mathbf{r}}(\mathbf{X})$ we define the (configuration and reaction dependent) set of admissible right velocities,

$$
\mathscr{K}_{\mathbf{v}}(\mathbf{X}, \mathbf{r}) \doteq\left\{\mathbf{v} \in \mathbb{R}^{2 n_{C}}: v_{n} \leqslant 0, \text { in } \mathscr{P}_{z} ; \quad v_{n}=0 \text { and } \sigma\left[r_{t}\right] v_{t} \leqslant 0, \text { in } \mathscr{P}_{s} ; \quad v_{n}=v_{t}=0, \text { in } \mathscr{P}_{d}\right\} .
$$


Having in mind (9), the (configuration and reaction dependent) set of admissible right reaction rates is defined by

$$
\mathscr{K}_{\mathbf{w}}(\mathbf{X}, \mathbf{r}) \doteq\left\{\mathbf{w} \in \mathbb{R}^{2 n_{C}}: w_{n}=w_{t}=0, \text { in } \mathscr{P}_{f} ; \quad w_{n} \leqslant 0,\left|w_{t}\right|+\mu w_{n} \leqslant 0, \text { in } \mathscr{P}_{z} ; \quad \sigma\left[r_{t}\right] w_{t}+\mu w_{n} \leqslant 0, \text { in } \mathscr{P}_{s}\right\}
$$

As done in (10) and (11), the dependency of some quantities on the contact particle $p$ is omitted in some circumstances, i.e. $v_{n}^{p}, v_{t}^{p}, w_{n}^{p}$ and $w_{t}^{p}$ are sometimes abbreviated to $v_{n}, v_{t}, w_{n}$ and $w_{t}$, respectively. The definitions of the sets $\mathscr{K}_{\mathbf{v}}(\mathbf{X}, \mathbf{r})$ and $\mathscr{K}_{\mathrm{w}}(\mathbf{X}, \mathbf{r})$ take into account the conditions that involve solely the kinematic variables $\mathbf{v}$ and solely the static variables $\mathbf{w}$. For known configurations and reactions, the right displacement and reaction rates have to satisfy other conditions than those expressed in the definitions of $\mathscr{K}_{\mathrm{v}}(\mathbf{X}, \mathbf{r})$ and $\mathscr{K}_{\mathbf{w}}(\mathbf{X}, \mathbf{r})$. Such conditions are complementarity conditions: $v_{n} w_{n}=0$ and $w_{t} v_{t}-\mu w_{n}\left|v_{t}\right|=0$ in $\mathscr{P}_{z}$ and $\left(\sigma\left[r_{t}\right] v_{t}\right)\left(\sigma\left[r_{t}\right] w_{t}+\mu w_{n}\right)=0$ in $\mathscr{P}_{s}$ that involve both velocity and reaction rates. These conditions may be written in the form [19]

$$
\mathbf{w} \cdot g(\mathbf{v})=0
$$

where the map $g: \mathbb{R}^{2 n_{C}} \rightarrow \mathbb{R}^{2 n_{C}}$ results from the maps $g^{p}: \mathbb{R}^{2} \rightarrow \mathbb{R}^{2}$ given, for each contact candidate particle $p$, by

$$
\begin{aligned}
& g^{p}\left(\mathbf{v}^{p}\right)=\left\{\begin{array}{c}
v_{n}^{p}-\mu\left|v_{t}^{p}\right| \\
v_{t}^{p}
\end{array}\right\} \text { for } p \in \mathscr{P}_{z}(\mathbf{X}, \mathbf{r}), \\
& g^{p}\left(\mathbf{v}^{p}\right)=\left\{\begin{array}{c}
v_{n}^{p}+\mu \sigma\left[r_{t}^{p}\right] v_{t}^{p} \\
v_{t}^{p}
\end{array}\right\} \quad \text { for } p \in \mathscr{P}_{s}(\mathbf{X}, \mathbf{r}), \\
& g^{p}\left(\mathbf{v}^{p}\right)=\left\{\begin{array}{l}
v_{n}^{p} \\
v_{t}^{p}
\end{array}\right\} \quad \text { for } p \in \mathscr{P}_{f}(\mathbf{X}) .
\end{aligned}
$$

\section{The study of the directionally linearized system}

\subsection{The directional instability eigenproblems}

In the neighborhood of the equilibrium state, and for admissible directions of the increments of the generalized coordinates and of the contact reactions, respectively $\delta \mathbf{X}(t)$ and $\delta \mathbf{r}(t)$,

$$
\mathbf{G}\left(\mathbf{X}^{0}\right) \delta \mathbf{X}(t) \in \mathscr{K}_{\mathbf{v}}(\mathbf{X}, \mathbf{r}), \quad \delta \mathbf{r}(t) \in \mathscr{K}_{\mathbf{w}}(\mathbf{X}, \mathbf{r}), \quad \delta \mathbf{r}(t) \cdot g\left(\mathbf{G}\left(\mathbf{X}^{0}\right) \delta \mathbf{X}(t)\right)=0,
$$

the equations of motion (7) have the following directionally linearized form

$$
\mathbf{M}\left(\mathbf{X}^{0}\right) \delta \ddot{\mathbf{X}}(t)+\mathbf{K}\left(\mathbf{X}^{0}, \mathbf{r}^{0}\right) \delta \mathbf{X}(t)=\mathbf{G}^{\mathrm{T}}\left(\mathbf{X}^{0}\right) \delta \mathbf{r}(t) .
$$

The tangent stiffness matrix $\mathbf{K} \in \mathbb{R}^{N \times N}$ has elastic contributions $\left(\mathbf{K}^{U}(\mathbf{X})\right)$, geometric stiffness contributions due to the constant external applied forces $\left(\mathbf{K}^{\Omega}(\mathbf{X}, \Lambda)\right)$, and contributions from obstacle curvature and contact reactions $\left(\mathbf{K}^{C}(\mathbf{X}, \mathbf{r})\right)[17,20]$

$$
\mathbf{K}=\mathbf{K}^{U}(\mathbf{X})+\mathbf{K}^{\Omega}(\mathbf{X}, \Lambda)+\mathbf{K}^{C}(\mathbf{X}, \mathbf{r}),
$$

where

$$
K_{i j}^{C}=-\sum_{p \in P_{c}}\left[\left(r_{n}^{p} \mathbf{n}^{p}+r_{t}^{p} \mathbf{t}^{p}\right) \frac{\partial^{2} \mathbf{x}^{p}}{\partial X_{i} \partial X_{j}}-\chi\left(r_{n}^{p} G_{t i}^{p} G_{t j}^{p}-r_{t}^{p} G_{n i}^{p} G_{t j}^{p}\right)\right]
$$

and $\mathbf{x}^{p}(\mathbf{X})$ is the position vector of particle $p$ in some fixed orthonormal reference frame. 
In [19] it is shown that

Proposition 1 (Directional instability of the linearized system). For $t$ in some right neighborhood of some instant $\tau(t \in[\tau, \tau+\Delta \tau[)$, there exist dynamic solutions of the form

$$
\delta \mathbf{X}(t)=\alpha(t) \mathbf{V} \in \mathbb{R}^{N}, \quad \delta \mathbf{r}(t)=\beta(t) \mathbf{w} \in \mathbb{R}^{2 n_{C}},
$$

where $\mathbf{V}$ and $\mathbf{w}$ define constant directions in the sets of right admissible displacement and reaction rates at the equilibrium state $\left(\mathbf{X}^{0}, \mathbf{r}^{0}\right)$, the function of time $\alpha$ is twice continuously differentiable, $\alpha$ and $\dot{\alpha}$ are non-negative and non-decreasing in the same interval, the initial values $\alpha(\tau) \geqslant 0, \dot{\alpha}(\tau) \geqslant 0$ are arbitrarily small, the function $\beta$ is continuous, non-negative and non-decreasing, if and only if there exists a number $\lambda \geqslant 0$ and two vectors $\mathbf{V}$ in $\mathbb{R}^{N}, \mathbf{V} \neq \mathbf{0}$ and $\mathbf{w}$ in $\mathbb{R}^{2 n_{C}}$ such that

$$
\begin{aligned}
& \left(\lambda^{2} \mathbf{M}+\mathbf{K}\right) \mathbf{V}=\mathbf{G}^{\mathrm{T}} \mathbf{w}, \\
& \mathbf{G V} \in \mathscr{K}_{\mathbf{v}}\left(\mathbf{X}^{0}, \mathbf{r}^{0}\right), \quad \mathbf{w} \in \mathscr{K}_{\mathbf{w}}\left(\mathbf{X}^{0}, \mathbf{r}^{0}\right), \quad \mathbf{w} \cdot g(\mathbf{G V})=0 .
\end{aligned}
$$

In these circumstances

$$
\beta(t)=\alpha(t)=\left\{\begin{array}{l}
\alpha(\tau) \cosh [\lambda(t-\tau)]+\left[\frac{\dot{\alpha}(\tau)}{\lambda}\right] \sinh [\lambda(t-\tau)], \text { if } \lambda>0, \\
\alpha(\tau)+\dot{\alpha}(\tau)(t-\tau), \text { if } \lambda=0,
\end{array}\right.
$$

and the equilibrium state $\left(\mathbf{X}^{0}, \mathbf{r}^{0}\right)$ of the linearized system is unstable by divergence.

The problem of the directional instability of the linearized system is formulated in (19) and (20) as a mixed explicit complementarity eigenproblem; inclusion or variational inequality formulations for the same problem were presented in [19]. Note that when a transformation of variables (2) is assumed to have been performed, so that the normal and tangential contact velocities are already generalized velocities of the system, the complementarity eigenproblems (19) and (20) may be equivalently written as

Find $\lambda \geqslant 0$ and $(\widetilde{\mathbf{V}}, \widetilde{\mathbf{W}}) \in \mathbb{R}^{N} \times \mathbb{R}^{N}$, with $\widetilde{\mathbf{V}} \neq \mathbf{0}$, such that

$$
\begin{aligned}
& \left(\lambda^{2} \widetilde{\mathbf{M}}+\widetilde{\mathbf{K}}\right) \widetilde{\mathbf{V}}=\widetilde{\mathbf{W}}, \\
& \widetilde{\mathbf{V}}=\left\{\begin{array}{c}
\widetilde{\mathbf{V}}_{F} \\
\mathbf{v}
\end{array}\right\} \neq \mathbf{0}, \quad \mathbf{v} \in \mathscr{K}_{\mathbf{v}}\left(\mathbf{X}^{0}, \mathbf{r}^{0}\right), \quad \widetilde{\mathbf{W}}=\left\{\begin{array}{c}
\mathbf{0} \\
\mathbf{w}
\end{array}\right\}, \quad \mathbf{w} \in \mathscr{K}_{\mathbf{w}}\left(\mathbf{X}^{0}, \mathbf{r}^{0}\right), \\
& \mathbf{w} \cdot g(\mathbf{v})=0,
\end{aligned}
$$

where $\widetilde{\mathbf{M}}=\mathbf{T}^{\mathrm{T}} \mathbf{M T}$ and $\widetilde{\mathbf{K}}=\mathbf{T}^{\mathrm{T}} \mathbf{K} \mathbf{T}$. Moreover, the dimension of the problem can be reduced by elimination of the two degrees of freedom of the stick particles $\left(\mathscr{P}_{d}\right)$ and of the normal degree of freedom of the particles in contact with non-vanishing reaction on the friction cone (the impending slip particles $\mathscr{P}_{s}$ ). Then we perform the transformation of variables

$$
\boldsymbol{\Xi} \doteq\left\{\begin{array}{c}
\boldsymbol{\Xi}_{f} \\
\boldsymbol{\Xi}_{s t} \\
\boldsymbol{\Xi}_{z n} \\
\boldsymbol{\Xi}_{z t}
\end{array}\right\}=\left\{\begin{array}{c}
\left\{\begin{array}{c}
\mathbf{V}_{F} \\
\mathbf{v}_{f}
\end{array}\right\} \\
\mathbf{S}_{s} \mathbf{v}_{s t} \\
-\mathbf{v}_{z n} \\
\mathbf{v}_{z t}
\end{array}\right\} \in \mathbb{R}^{N^{*}}, \quad \boldsymbol{\Psi} \doteq\left\{\begin{array}{l}
\boldsymbol{\Psi}_{f} \\
\boldsymbol{\Psi}_{s t} \\
\boldsymbol{\Psi}_{z n} \\
\boldsymbol{\Psi}_{z t}
\end{array}\right\}=\left\{\begin{array}{c}
\left\{\begin{array}{l}
\mathbf{0} \\
\mathbf{0}
\end{array}\right\} \\
\mathbf{S}_{s} \mathbf{w}_{s t}-\mu_{s} \mathbf{w}_{s n} \\
-\mathbf{w}_{z n} \\
\mathbf{w}_{z t}
\end{array}\right\} \in \mathbb{R}^{N^{*}}
$$

where $\mathbf{S}_{s}=-\operatorname{diag}\left(\sigma\left[r_{t}^{0 p}\right], p \in \mathscr{P}_{s}\left(\mathbf{X}^{0}, \mathbf{r}^{0}\right)\right)$ and $N^{*}=n_{F}+n_{f}+n_{s}+2 n_{z}$ is the number of degrees of freedom that may be (right) active, which includes all the free degrees of freedom $(F)$ and those of the contact 
candidate particles that are currently free $(f)$, plus the tangential degrees of freedom $(t)$ of the particles $(s)$ in impending slip, as well as both degrees of freedom ( $n$ and $t$ ) of the particles in contact with zero reaction $(z)$. The eigenproblem (19) and (20) is then

Find $\lambda \geqslant 0$ and $(\boldsymbol{\Xi}, \boldsymbol{\Psi}) \in \mathbb{R}^{N^{*}} \times \mathbb{R}^{N^{*}}$, with $\boldsymbol{\Xi} \neq \mathbf{0}$, such that

$$
\begin{aligned}
& \left(\lambda^{2} \mathbf{M}^{*}+\mathbf{K}^{*}\right) \boldsymbol{\Xi}=\boldsymbol{\Psi}, \\
& \boldsymbol{\Psi}_{f}=\mathbf{0}, \quad \mathbf{0} \leqslant \boldsymbol{\Xi}_{s t} \perp \boldsymbol{\Psi}_{s t} \geqslant \mathbf{0}, \quad \mathbf{0} \leqslant \boldsymbol{\Xi}_{z n} \perp \boldsymbol{\Psi}_{z n} \geqslant \mathbf{0}, \\
& \psi_{z t}^{p} \in-\mu_{z} \psi_{z n}^{p} \sigma\left[\Xi_{z t}^{p}\right], \quad p \in \mathscr{P}_{z} .
\end{aligned}
$$

The matrices $\mathbf{M}^{*}$ and $\mathbf{K}^{*}$ are linear pencils of matrices in the coefficient of friction at the particles in impending slip,

$$
\mathbf{M}^{*}=\mathbf{M}^{*}\left(\mu_{s}\right)=\mathbf{M}_{0}-\mu_{s} \mathbf{M}_{1}, \quad \mathbf{K}^{*}=\mathbf{K}^{*}\left(\mu_{s}\right)=\mathbf{K}_{0}-\mu_{s} \mathbf{K}_{1} .
$$

The structure of the above non-symmetric pencils of matrices is the same and is illustrated below for the mass matrix

$$
\begin{aligned}
\mathbf{M}_{0}= & {\left[\begin{array}{cccc}
\widetilde{\mathbf{M}}_{f, f} & \widetilde{\mathbf{M}}_{f, s t} \mathbf{S}_{s} & -\widetilde{\mathbf{M}}_{f, z n} & \widetilde{\mathbf{M}}_{f, z t} \\
\mathbf{S}_{s} \widetilde{\mathbf{M}}_{s t, f} & \mathbf{S}_{s} \widetilde{\mathbf{M}}_{s t, s t} \mathbf{S}_{s} & -\mathbf{S}_{s} \widetilde{\mathbf{M}}_{s t, z n} & \mathbf{S}_{s} \widetilde{\mathbf{M}}_{s t, z t} \\
-\widetilde{\mathbf{M}}_{z n, f} & -\widetilde{\mathbf{M}}_{z n, s t} \mathbf{S}_{s} & \widetilde{\mathbf{M}}_{z n, z n} & -\widetilde{\mathbf{M}}_{z n, z t} \\
\widetilde{\mathbf{M}}_{z t, f} & \widetilde{\mathbf{M}}_{z t, s t} \mathbf{S}_{s} & -\widetilde{\mathbf{M}}_{z t, z n} & \widetilde{\mathbf{M}}_{z t, z t}
\end{array}\right], } \\
\mathbf{M}_{1}= & {\left[\begin{array}{cccc}
\mathbf{0} & \mathbf{0} & \mathbf{0} & \mathbf{0} \\
\widetilde{\mathbf{M}}_{s n, f} & \widetilde{\mathbf{M}}_{s n, s t} \mathbf{S}_{s} & -\widetilde{\mathbf{M}}_{s n, z n} & \widetilde{\mathbf{M}}_{s n, z t} \\
\mathbf{0} & \mathbf{0} & \mathbf{0} & \mathbf{0} \\
\mathbf{0} & \mathbf{0} & \mathbf{0} & \mathbf{0}
\end{array}\right] . }
\end{aligned}
$$

\subsection{Equivalence to a set of generalized linear eigenproblems together with some inequalities}

It is important to observe that the resolution of the eigenproblem (19) and (20) or (26)-(28) is equivalent to the resolution of a set of classical generalized linear eigenproblems, together with the verification of some inequalities. These various linear eigenproblems are obtained by considering all possible combinations of near future states of the contact candidate particles. Actually, since the particles in $\mathscr{P}_{f}\left(\mathbf{X}_{0}\right)$ (currently free) and in $\mathscr{P}_{d}\left(\mathbf{X}_{0}, \mathbf{r}_{0}\right)$ (currently stuck) will remain so in the near future, only the combinations of possible near future states of the particles in $\mathscr{P}_{z}\left(\mathbf{X}_{0}, \mathbf{r}_{0}\right)$ and $\mathscr{P}_{s}\left(\mathbf{X}_{0}, \mathbf{r}_{0}\right)$ need to be considered. The total number of classical generalized linear eigenproblems that may be constructed in this manner is thus $2^{n_{s}} \times 4^{n_{z}}$, because a contact particle in $\mathscr{P}_{s}$ has two possible near future evolutions (stick and positive or negative slip, opposite to the static tangential reaction), while a particle in $\mathscr{P}_{z}$ has four possible near future evolutions (free, positive slip, negative slip and stick). Assuming now that one combination of the above near future evolutions holds, the transformations of variables

$$
\widetilde{\mathbf{V}}=\left\{\begin{array}{c}
\mathbf{V}_{F} \\
\mathbf{v}
\end{array}\right\}=\left\{\begin{array}{c}
\mathbf{v}_{\text {free }} \\
\mathbf{v}_{\text {slip } t} \\
\mathbf{v}_{\text {slip } n} \\
\mathbf{v}_{\text {stick }}
\end{array}\right\}=\left\{\begin{array}{c}
\boldsymbol{\Xi}_{\text {free }} \\
\mathbf{S}_{\text {slip }} \boldsymbol{\Xi}_{\text {slip } t} \\
\mathbf{0} \\
\mathbf{0}
\end{array}\right\}, \quad\left\{\begin{array}{c}
\boldsymbol{\Xi}_{\text {free }} \\
\boldsymbol{\Xi}_{\text {slip } t}
\end{array}\right\}=\boldsymbol{\Xi} \in \mathbb{R}^{N^{\prime}},
$$




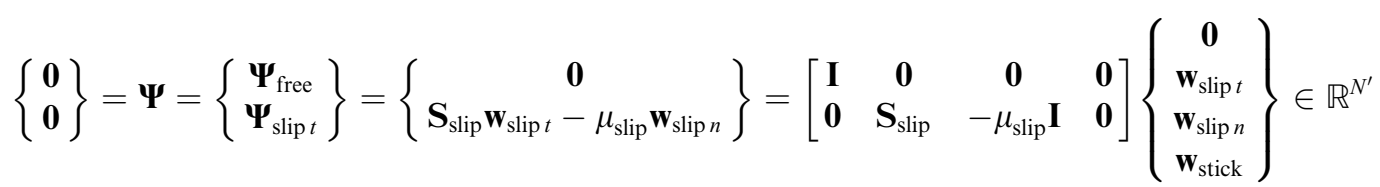

are applied. In this manner, both degrees of freedom of the particles in contact with reaction strictly inside the friction cone (the stick particles), as well as the normal (n) degrees of freedom of the slip particles are eliminated, and the generalized linear eigenproblem

$$
\left(\lambda^{2} \mathbf{M}^{\prime}+\mathbf{K}^{\prime}\right) \boldsymbol{\Xi}=\mathbf{0}
$$

is obtained, where $\mathbf{M}^{\prime}$ and $\mathbf{K}^{\prime}$ have the structure

$$
\mathbf{M}^{\prime}=\left[\begin{array}{cc}
\widetilde{\mathbf{M}}_{\text {free,free }} & \widetilde{\mathbf{M}}_{\text {free,slip } t} \mathbf{S}_{\text {slip }} \\
\mathbf{S}_{\text {slip }} \widetilde{\mathbf{M}}_{\text {slip } t \text {,free }} & \mathbf{S}_{\text {slip }} \widetilde{\mathbf{M}}_{\text {slip } t, \text { slip } t} \mathbf{S}_{\text {slip }}
\end{array}\right]-\mu_{\text {slip }}\left[\begin{array}{cc}
\mathbf{0} & \mathbf{0} \\
\widetilde{\mathbf{M}}_{\text {slip } n, \text { free }} & \widetilde{\mathbf{M}}_{\text {slip } n, \text { slip } t} \mathbf{S}_{\text {slip }}
\end{array}\right] .
$$

Matrices $\mathbf{M}^{\prime}$ and $\mathbf{K}^{\prime}$ are, respectively, the effective mass matrix and the effective stiffness matrix corresponding to the assumed combination of the near future evolutions of the contact particles. The $p$ diagonal component $s^{p}$ of the diagonal matrix $\mathbf{S}_{\text {slip }}$, refers now to a particle that is assumed to slip: $s^{p}$ has necessarily the value $-\sigma\left[r_{t}^{0 p}\right]$ if $p \in \mathscr{P}_{s}\left(\mathbf{X}_{0}, \mathbf{r}_{0}\right)$, and has one of the values +1 or -1 , if $p \in \mathscr{P}_{z}\left(\mathbf{X}_{0}, \mathbf{r}_{0}\right)$. In order that the solutions of (34) do solve the original eigenproblem (19) and (20), or (22)-(24), or (26)-(28), the following inequalities must be satisfied, in addition to the equalities taken into account by the construction (32) and (33) [recall the inequalities in the admissible sets (10) and (11)]:

for the particles $p$ in $\mathscr{P}_{z}$ that were assumed to become

$$
\text { free: } \quad-v_{n}^{p} \geqslant 0
$$

slip: $\quad s^{p} v_{t}^{p} \geqslant 0, \quad-w_{n}^{p} \geqslant 0$,

stick: $\quad-\left|w_{t}^{p}\right|-\mu w_{n}^{p} \geqslant 0$,

for the particles $p$ in $\mathscr{P}_{s}$ that were assumed to become

slip: $\quad s^{p} v_{t}^{p} \geqslant 0$,

stick: $\quad s^{p} w_{t}^{p}-\mu w_{n}^{p} \geqslant 0$.

\subsection{The occurrence of directional instabilities: sufficient conditions and necessary conditions}

The set of (homogeneous) equations and conditions (22)-(24) or (26)-(28) has always the trivial solution. In what conditions is that trivial solution unique? To study this it is convenient to rewrite (28) in the form

$$
\begin{aligned}
& \boldsymbol{\Psi}_{z t}=\mathbf{w}_{z t}=\mu_{z} \mathbf{S}_{z} \mathbf{w}_{z n}=-\mu_{z} \mathbf{S}_{z} \boldsymbol{\Psi}_{z n}, \\
& \mathbf{S}_{z}=\operatorname{diag}\left(s^{p}\right), \quad s^{p} \in[-1,+1], \quad p \in \mathscr{P}_{z}, \\
& \Xi_{z t}^{p}>0 \Rightarrow s^{p}=+1 ; \quad \Xi_{z t}^{p}<0 \Rightarrow s^{p}=-1, \quad p \in \mathscr{P}_{z},
\end{aligned}
$$

and the governing equation (26) in the form

$$
\left(\lambda^{2} \mathbf{M}^{*}+\mathbf{K}^{*}\right)\left\{\begin{array}{c}
\boldsymbol{\Xi}_{f} \\
\boldsymbol{\Xi}_{s t} \\
\boldsymbol{\Xi}_{z n} \\
\boldsymbol{\Xi}_{z t}
\end{array}\right\}=\left[\begin{array}{cccc}
\mathbf{I} & \mathbf{0} & \mathbf{0} & \mathbf{0} \\
\mathbf{0} & \mathbf{I} & \mathbf{0} & \mathbf{0} \\
\mathbf{0} & \mathbf{0} & \mathbf{I} & \mathbf{0} \\
\mathbf{0} & \mathbf{0} & -\mu_{z} \mathbf{S}_{z} & \mathbf{I}
\end{array}\right]\left\{\begin{array}{c}
\mathbf{0} \\
\boldsymbol{\Psi}_{s t} \\
\boldsymbol{\Psi}_{z n} \\
\mathbf{0}
\end{array}\right\}
$$


The coefficient matrix of the right-hand side is non-singular, so that the previous algebraic equation is equivalent to

$$
\left\{\begin{array}{c}
\mathbf{0} \\
\boldsymbol{\Psi}_{s t} \\
\boldsymbol{\Psi}_{z n} \\
\mathbf{0}
\end{array}\right\}=\left[\begin{array}{cccc}
\mathbf{I} & \mathbf{0} & \mathbf{0} & \mathbf{0} \\
\mathbf{0} & \mathbf{I} & \mathbf{0} & \mathbf{0} \\
\mathbf{0} & \mathbf{0} & \mathbf{I} & \mathbf{0} \\
\mathbf{0} & \mathbf{0} & \mu_{z} \mathbf{S}_{z} & \mathbf{I}
\end{array}\right]\left(\lambda^{2} \mathbf{M}^{*}+\mathbf{K}^{*}\right)\left\{\begin{array}{c}
\mathbf{\Xi}_{f} \\
\boldsymbol{\Xi}_{s t} \\
\boldsymbol{\Xi}_{z n} \\
\mathbf{\Xi}_{z t}
\end{array}\right\}
$$

where the components of the vectors involved satisfy

$$
\begin{aligned}
& \Xi_{s t}^{p} \psi_{s t}^{p}=0, \\
& \Xi_{z n}^{p} \psi_{z n}^{p}=0 .
\end{aligned}
$$

Then, if all the pencils of matrices constructed from $\lambda^{2} \mathbf{M}^{*}+\mathbf{K}^{*}$ with $\lambda \geqslant 0$ as indicated in the next proposition are of class $P[7,25]$, the only solution is $(\boldsymbol{\Xi}, \Psi)=(\mathbf{0}, \mathbf{0})$. The next proposition gives then a sufficient condition for the linearized system not to be directionally unstable.

Proposition 2 (Uniqueness). A sufficient condition for the set of equations and conditions of the directional instability problem (26)-(28) to have only the trivial solution is that, for $\lambda \geqslant 0$ and for any $\mathbf{S}_{z}=\operatorname{diag}\left(s^{p}\right), s^{p} \in[-1,+1], p \in \mathscr{P}_{z}$, the non-symmetric matrix below is a P matrix:

$$
\left[\begin{array}{cccc}
\mathbf{I} & \mathbf{0} & \mathbf{0} & \mathbf{0} \\
\mathbf{0} & \mathbf{I} & \mathbf{0} & \mathbf{0} \\
\mathbf{0} & \mathbf{0} & \mathbf{I} & \mathbf{0} \\
\mathbf{0} & \mathbf{0} & \mu_{z} \mathbf{S}_{z} & \mathbf{I}
\end{array}\right]\left[\lambda^{2} \mathbf{M}^{*}\left(\mu_{s}\right)+\mathbf{K}^{*}\left(\mu_{s}\right)\right]
$$

In the linear elasticity case this holds if $\mu_{s}$ and $\mu_{z}$ are sufficiently small and the obstacle curvature $\chi$ is sufficiently small when positive.

An interval matrix is a matrix whose components are intervals (for further details see [22,26]). When this definition is used, the usual matrices, whose components are real numbers, are called point matrices. Since each of the real numbers $s^{p}, p \in \mathscr{P}_{z}$, may assume an arbitrary value in the interval $[-1,+1]$, the set of all matrices obtained from (44) when all $s^{p}$ run over all their admissible values may be considered an interval matrix, in which the generic point matrix $\mathbf{S}_{z}=\operatorname{diag}\left(s^{p}, p \in \mathscr{P}_{z}\right), s^{p} \in[-1,+1]$ gives rise to a $n_{z} \times n_{z}$ diagonal interval matrix $\mathbb{S}_{z}$, whose diagonal components are the intervals $[-1,+1]\left(\mathbb{S}_{z}=\right.$ $\operatorname{diag}([-1,+1]))$. With these definitions the uniqueness condition in Proposition 2 can be re-phrased as: the directional instability problem (26)-(28) has only the trivial solution if, for any $\lambda \geqslant 0$, the interval matrix pencil

$$
\mathbb{\square}_{z}\left(\mu_{z}\right)\left(\lambda^{2} \mathbf{M}^{*}\left(\mu_{s}\right)+\mathbf{K}^{*}\left(\mu_{s}\right)\right)
$$

is of class $P$, where

$$
\mathbb{\nabla}_{z}\left(\mu_{z}\right)=\left[\begin{array}{cccc}
\mathbf{I} & \mathbf{0} & \mathbf{0} & \mathbf{0} \\
\mathbf{0} & \mathbf{I} & \mathbf{0} & \mathbf{0} \\
\mathbf{0} & \mathbf{0} & \mathbf{I} & \mathbf{0} \\
\mathbf{0} & \mathbf{0} & \mu_{z} \mathbb{S}_{z} & \mathbf{I}
\end{array}\right] .
$$

Of course that an interval matrix is said to be of class $P$ if and only if all the point matrices in the interval matrix are of class $P$. We can also say that the directional instability problem (26)-(28) has only the trivial solution if, for any $\lambda \geqslant 0$, the matrix pencil $\lambda^{2} \mathbf{M}^{*}\left(\mu_{s}\right)+\mathbf{K}^{*}\left(\mu_{s}\right)$ is of class $P$ and $\mu_{z}$ is sufficiently small. We observe that, for the pencil $\lambda^{2} \mathbf{M}^{*}\left(\mu_{s}\right)+\mathbf{K}^{*}\left(\mu_{s}\right)$ to be of class $P$ it is not sufficient that the matrices $\mathbf{M}^{*}$ and $\mathbf{K}^{*}$ 
be of class $P$, because the sum of two matrices of class $P$ is not, in general, a matrix of class $P$. However, if the mass matrix $\widetilde{\mathbf{M}}$ is a diagonal (necessarily $P D$ ) matrix then the matrix $\mathbf{M}^{*}\left(\mu_{s}\right)$ is also diagonal and $P D$, and its sum with $\mathbf{K}^{*}$ is also $P$ if the latter matrix is $P$. A matrix $\mathbf{A}$ is positive definite $(P D)$ if and only if $\mathbf{x}^{\mathrm{T}} \mathbf{A} \mathbf{x}>0$ for any $\mathbf{x}$ different from $\mathbf{0}$. We conclude that when the matrix $\widetilde{\mathbf{M}}$ is diagonal, the matrix $\mathbf{K}^{*}$ is $P$, and $\mu_{z}$ is sufficiently small, then the linearized system is not directionally unstable.

Proposition 2 and subsequent statements are expressed in terms of the property $P$, which takes into account the non-symmetry of the matrices involved. Other conditions that do not take into account that non-symmetric (i.e. non-associative) character of the Coulomb friction law may be deduced [5,19,29]: doing the inner product of (26) with $\boldsymbol{\Xi}$, it is easy to conclude that a necessary condition for the occurrence of $a$ directional instability of the linearized system is that

the matrix $\mathbf{M}^{*}\left(\mu_{s}\right)$ is not $P D$ or the matrix $\mathbf{K}^{*}\left(\mu_{s}\right)$ is not $P D$.

However, the conditions expressed in terms of the property $P$ are sharper, in the sense that they yield less conservative (higher) estimates for the coefficient of friction of the transition to directional instability.

On the other hand, a sufficient condition for the occurrence of a directional instability for the linearized system is the following [19]

If there is a $\lambda \geqslant 0$ and $\boldsymbol{\Xi} \in \mathbb{R}^{N^{*}}$, with $\boldsymbol{\Xi} \neq \mathbf{0}$, such that

$$
\begin{aligned}
& \left(\lambda^{2} \mathbf{M}^{*}+\mathbf{K}^{*}\right) \boldsymbol{\Xi}=\mathbf{0}, \\
& \boldsymbol{\Xi}_{s t} \geqslant \mathbf{0}, \\
& \boldsymbol{\Xi}_{z n} \geqslant \mathbf{0},
\end{aligned}
$$

then the linearized system is directionally unstable.

Note that conditions (48)-(50) are a particular case of (26)-(28) that results from a priori assuming that the solution satisfies: (i) $\boldsymbol{\Psi}_{s t}=\mathbf{0}$, i.e. the reactions of the contact particles in impending slip (the $s$ particles) remain on the boundary of the friction cone, which means that those particles remain in impending slip or do initiate sliding and (ii) $\boldsymbol{\Psi}_{z n}=\mathbf{0}$, i.e. the reactions of the contact particles in contact with zero reaction (the $z$ particles) will remain equal to 0 , which means that those particles remain in contact with zero reaction or loose contact. Whenever they exist, the solutions of (48)-(50) will be called all-slip solutions. Note that (26)-(28) is a mixed complementarity-inclusion eigenproblem while (48)-(50) is a much simpler problem: a classical generalized linear eigenproblem with a set of inequalities (49) and (50) to be verified a posteriori.

In numerical computations of quasi-static evolutions it is observed that the necessary condition (47) is satisfied (significantly) earlier than the sufficient condition (48)-(50) [19]. The reason that motivates the computational study of the original problem (19) and (20) (or (26)-(28)) is the existence of examples, like the one presented in the next subsection, in which the necessary condition (47) is satisfied, the sufficient condition (48)-(50) is never satisfied, and there are solutions to the problem (19) and (20) or (26)-(28) that do not involve the slip of all the contact particles in impending slip, i.e., solutions that do not satisfy the sufficient condition (48)-(50).

\subsection{An example of a non-all-slip instability mode}

The model schematically represented in Fig. 2 consists of a four node bilinear finite element made of a linear elastic isotropic material in a plane stress state. The two relevant non-dimensional parameters are the aspect ratio $\beta=b / a$ and the Poisson ratio of the material $v$. We assume that, at the equilibrium configuration, both nodes $C$ and $D$ are in impending slip to the left $\left(\mathscr{P}_{z}=\emptyset\right)$. The eigenproblem (26)-(28) at the transition value $\lambda=0$ is governed by 


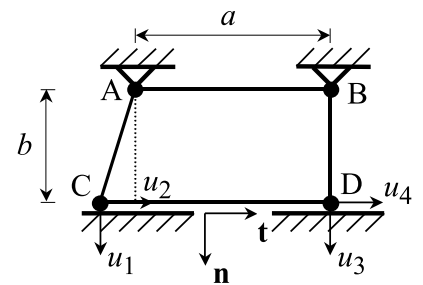

Fig. 2. Bilinear finite element with two nodes in impending slip to the left.

$$
\begin{aligned}
& {\left[\begin{array}{ll}
4 \beta+\frac{2(1-v)}{\beta}-\mu \frac{3}{2}(1+v) & -4 \beta+\frac{1-v}{\beta}-\mu \frac{3}{2}(1-3 v) \\
-4 \beta+\frac{1-v}{\beta}+\mu \frac{3}{2}(1-3 v) & 4 \beta+\frac{2(1-v)}{\beta}+\mu \frac{3}{2}(1+v)
\end{array}\right]\left\{\begin{array}{l}
\Xi_{s t}^{C} \\
\Xi_{s t}^{D}
\end{array}\right\}=\left\{\begin{array}{l}
\psi_{s t}^{C} \\
\psi_{s t}^{D}
\end{array}\right\},} \\
& \mathbf{0} \leqslant\left\{\begin{array}{l}
\Xi_{s t}^{C} \\
\Xi_{s t}^{D}
\end{array}\right\} \perp\left\{\begin{array}{l}
\psi_{s t}^{C} \\
\psi_{s t}^{D}
\end{array}\right\} \geqslant \mathbf{0} .
\end{aligned}
$$

where $\left(\left(\Xi_{s t}^{C}, \Xi_{s t}^{D}\right),\left(\psi_{s t}^{C}, \psi_{s t}^{D}\right)\right) \in \mathbb{R}^{2} \times \mathbb{R}^{2},\left(\Xi_{s t}^{C}, \Xi_{s t}^{D}\right) \neq(0,0)$, and the friction coefficient $\mu \geqslant 0$ at that transition is also an unknown. Fig. 3 represents the regions, in the parameter plane $(\beta, v)$, for the occurrence of the two possible instability modes that solve (51) and (52): SLIP-STICK (left slip of node $C$ and stick of node $D$ ) and SLIP-SLIP (left slip of both the contact nodes $C$ and $D$ ). These two regions are separated by a curve defined by the equation $5 v^{2}-8\left(1+2 \beta^{2}\right) v+3=0$. For $(\beta, v)$ on this curve $\lim _{\beta \rightarrow+\infty} v=0$, which means that the horizontal coordinate axis is an asymptote. For $(\beta, v)$ in the region of Fig. 3 below the curve, the onset of SLIP-STICK directional instability occurs for the coefficient of friction

$$
\mu=\frac{2 \beta+\frac{1-v}{\beta}}{\frac{3}{4}(1+v)},
$$

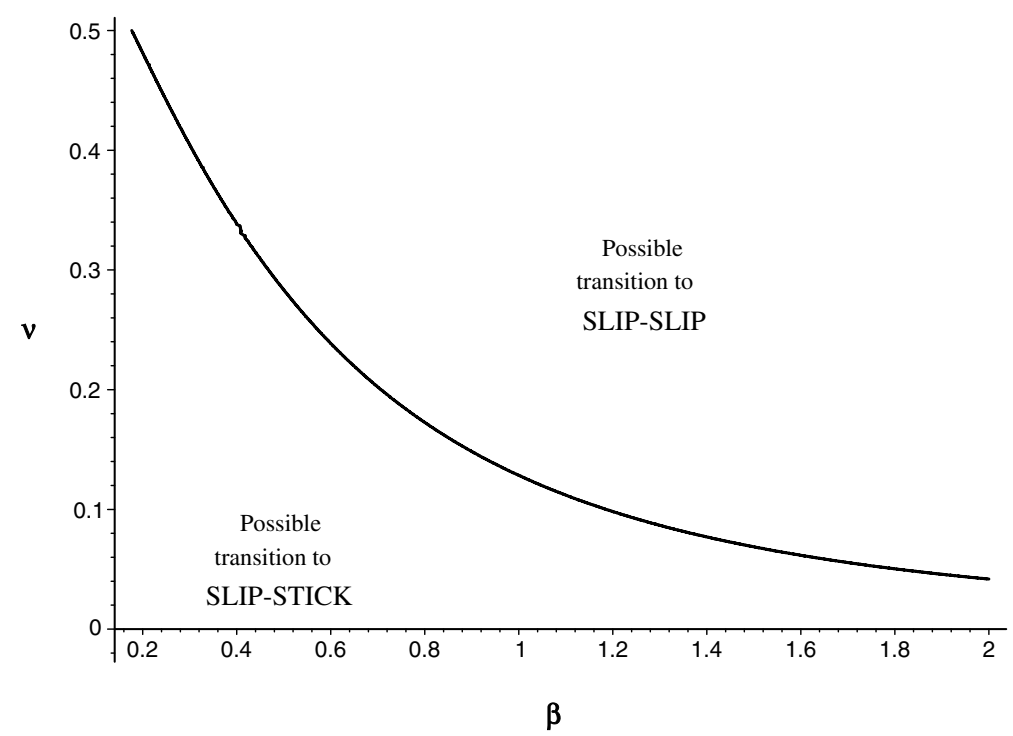

Fig. 3. Regions of the space of parameters $\nu-\beta$ for the occurrence of SLIP-SLIP or SLIP-STICK instability modes. 
and there is no possibility of transition to the SLIP-SLIP kind of instability. For $(\beta, v)$ in the region of Fig. 3 above the curve, the onset of SLIP-SLIP directional instability occurs for the coefficient of friction

$$
\mu=\sqrt{\frac{8+\frac{1-v}{\beta^{2}}}{6 v}}
$$

and the STICK-SLIP kind of directional instability is not possible. For parameters $(\beta, v)$ on the curve of Fig. 3, the two instability modes of the neighboring regions coalesce in a degenerate mode with $\Xi_{s t}^{D}=\psi_{s t}^{D}=0$. But the main conclusion to extract from this example is that in the parameter region $(\beta, v)$ below the curve of Fig. 3 there is no all-slip (SLIP-SLIP) solution satisfying the sufficient condition (48)-(50), but the instability eigenproblem (26)-(28) does have a SLIP-STICK solution of the type represented in Fig. 2, for values of $\mu$ greater than the one given in (53).

\section{Two mixed complementarity eigenproblems and their numerical solution}

\subsection{The problems MCEIP- $\lambda^{2}$ and MCEIP- $\mu$}

In the case of $\mathscr{P}_{z}=\emptyset$ the problem (26)-(28) leads to the mixed complementarity eigenproblem in $\lambda^{2}$ $\left(M C E I P-\lambda^{2}\right)$

Find $\lambda \geqslant 0$ and $(\boldsymbol{\Xi}, \boldsymbol{\Psi}) \in \mathbb{R}^{N^{*}} \times \mathbb{R}^{N^{*}}$, with $\boldsymbol{\Xi} \neq \mathbf{0}$, such that

$$
\begin{aligned}
& \left(\lambda^{2} \mathbf{M}^{*}+\mathbf{K}^{*}\right) \boldsymbol{\Xi}=\boldsymbol{\Psi}, \\
& \boldsymbol{\Psi}_{f}=\mathbf{0}, \quad \mathbf{0} \leqslant \boldsymbol{\Xi}_{s t} \perp \boldsymbol{\Psi}_{s t} \geqslant \mathbf{0},
\end{aligned}
$$

where now the vectors and matrices $\boldsymbol{\Xi}, \boldsymbol{\Psi}, \mathbf{M}^{*}$ and $\mathbf{K}^{*}$ have no contributions from the empty set $\mathscr{P}_{z}$. Hence

$$
\mathbf{K}^{*}=\left[\begin{array}{cc}
\widetilde{\mathbf{K}}_{f, f} & \widetilde{\mathbf{K}}_{f, s t} \mathbf{S}_{s} \\
\mathbf{S}_{s} \widetilde{\mathbf{K}}_{s t, f} & \mathbf{S}_{s} \widetilde{\mathbf{K}}_{s t, s t} \mathbf{S}_{s}
\end{array}\right]-\mu\left[\begin{array}{cc}
\mathbf{0} & \mathbf{0} \\
\widetilde{\mathbf{K}}_{s n, f} & \widetilde{\mathbf{K}}_{s n, s t} \mathbf{S}_{s}
\end{array}\right],
$$

$\mathbf{M}^{*}$ has a similar structure and $\mu=\mu_{s}$.

A problem related to the above $M C E I P-\lambda^{2}$ (55) and (56) consists of computing the coefficient of friction and the associated mode shapes that correspond to the transition eigenvalue $\lambda=0$, for a given equilibrium state $\left(\mathbf{X}^{0}, \mathbf{r}^{0}\right)$. Substituting this condition in (55), leads to a mixed complementarity eigenproblem in $\mu$ $(M C E I P-\mu)$ :

Find $\mu \geqslant 0$ and $(\boldsymbol{\Xi}, \boldsymbol{\Psi}) \in \mathbb{R}^{N^{*}} \times \mathbb{R}^{N^{*}}$, with $\boldsymbol{\Xi} \neq \mathbf{0}$, such that

$$
\begin{aligned}
& \left(\mathbf{K}_{0}-\mu \mathbf{K}_{1}\right) \boldsymbol{\Xi}=\boldsymbol{\Psi}, \\
& \boldsymbol{\Psi}_{f}=\mathbf{0}, \quad \mathbf{0} \leqslant \boldsymbol{\Xi}_{s t} \perp \boldsymbol{\Psi}_{s t} \geqslant \mathbf{0},
\end{aligned}
$$

where $\mu=\mu_{s}$.

\subsection{Numerical solution as mixed complementarity problems}

It is possible to rewrite the non-linear eigenproblems $M C E I P-\lambda^{2}$ and $M C E I P-\mu$ as mixed non-linear complementarity problems ( $M C P S$ ), which are then solved with the algorithm $P A T H$ [8]. This algorithm is based on a non-classic Newton method that builds a piecewise linear path and does a line search along that path. The transformation into a $M C P$ is achieved by taking the eigenvalue ( $\lambda^{2}$ or $\mu$ ) as an additional nonnegative variable, which is complementary to another non-negative variable $(\gamma)$, used in a normalizing 
constraint equation that involves the eigenvector $\Xi$. As in the previous subsection, only the case of $\mathscr{P}_{z}=\emptyset$ is considered.

In this manner the eigenproblem $M C E I P-\mu$ defined in (58) and (59) is transformed in the following mixed complementarity problem $(M C P-\mu)$ :

Find $(\boldsymbol{\Xi}, \mu) \in \mathbb{R}^{N^{*}+1}$ and $(\boldsymbol{\Psi}, \gamma) \in \mathbb{R}^{N^{*}+1}$, such that

$$
\begin{aligned}
& \left(\mathbf{K}_{0}-\mu \mathbf{K}_{1}\right) \boldsymbol{\Xi}=\boldsymbol{\Psi}, \\
& \mathbf{e}^{\mathrm{T}} \boldsymbol{\Xi}_{s t}=c+\gamma, \\
& \boldsymbol{\Psi}_{f}=\mathbf{0}, \\
& \mathbf{0} \leqslant\left\{\begin{array}{c}
\boldsymbol{\Xi}_{s t} \\
\mu
\end{array}\right\} \perp\left\{\begin{array}{c}
\boldsymbol{\Psi}_{s t} \\
\gamma
\end{array}\right\} \geqslant \mathbf{0},
\end{aligned}
$$

where the coefficient matrix is (57), $\mathbf{e}$ is a vector of dimension $n_{s}$ with all components equal to $1, c$ is an arbitrary positive real number that normalizes the mode shape and $N^{*}=n_{F}+n_{f}+n_{s}\left(n_{z}=0\right)$.

Note that if $(\boldsymbol{\Xi}, \mu)$ and $(\boldsymbol{\Psi}, \gamma)$ solve the $M C P-\mu(60)-(63)$, then $\mathbf{e}^{\mathrm{T}} \boldsymbol{\Xi}_{s t}=c+\gamma$ for some $c>0$, so that $\mathbf{e}^{\mathrm{T}} \boldsymbol{\Xi}_{s t}>0, \boldsymbol{\Xi}_{s t} \neq 0, \boldsymbol{\Xi} \neq 0$, and $\boldsymbol{\Xi}, \boldsymbol{\Psi}$ and $\mu$ solve the MCEIP- $\mu$ (58) and (59). The proof that any solution to $M C E I P-\mu$ (58) and (59) also solves $M C P-\mu(60)-(63)$ with $\gamma=0$ can be found in [31], where the analogous transformation of $M C E I P-\lambda^{2}$ into a $M C P$ is also discussed.

\section{Numerical examples by the finite element method}

\subsection{Finite element discretizations of a tribology experiment}

The first example is a model of tribology experiments done by Progri, Villechaise and Zeghloul [32,40] on rectangular polyurethane blocks sliding on an araldite base which may be considered a rigid obstacle. The elastic block is discretized with non-uniform meshes of 530, 776 and 1890 linear P1 finite elements that have, respectively 41, 65 and 161 contact candidate nodes. The block is assumed to be in a state of plane stress. Its elastic properties are: modulus of elasticity $=5 \mathrm{MPa}$, Poisson's ratio $=0.48$. The geometric parameters are the length $L=80 \mathrm{~mm}$ and the height $H=40 \mathrm{~mm}$ of the blocks. The density of the material is $1.2 \mathrm{~kg} / \mathrm{dm}^{3}$. The block is submitted to a quasi-static loading consisting first of prescribed displacements on the side $C D$ (see Fig. 4), which is symmetrically pressed against the obstacle until the resultant of the

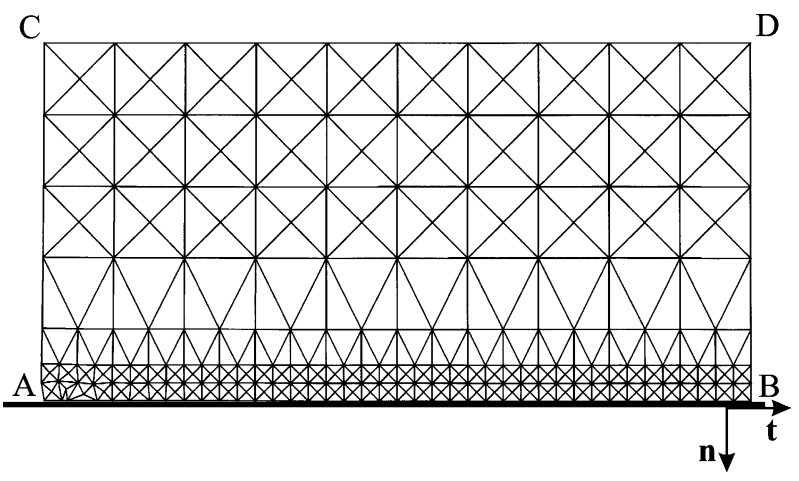

Fig. 4. An instability mode at the onset of instability for a mesh of 530 elements and $\mu=57.653929$ (solution of $M C E I P-\mu$ ). 
normal reactions on side $A B$ is $-55 \mathrm{~N}$. Then the loading proceeds by prescribing an horizontal motion of the side $C D$ towards the left $[32,40]$. In this tangential loading phase, the successive equilibrium states have a growing region of nodes in impending slip spreading from right to left.

This example was studied earlier in [19], also by the finite element method, but using only the necessary (47) and the sufficient (48)-(50) conditions for directional instability. For a coefficient of friction $\mu=1.1$ close to the experimentally observed value, the numerical results showed that the necessary condition (47) was satisfied very early along the tangential loading process; however, for all the successive equilibrium configurations of the block along that tangential loading, the sufficient condition (48)-(50), that involves slip of all nodes in impending slip, could never be satisfied. Our objective is thus to check if, after the necessary condition is satisfied, there exist instability modes of a type different from the all-slip modes of the sufficient condition (48)-(50).

With this purpose, we search first for solutions to the $M C E I P-\mu$ at the equilibrium states obtained with $\mu=1.1$, along the tangential loading of the block, i.e. we search for the values of the coefficient of friction corresponding to the onset $(\lambda=0)$ of instability in that equilibrium configuration. It is found that the values of $\mu$ that solve the $M C E I P-\mu$ are very large and decrease with the increase of the number of nodes in impending slip in the successive equilibrium configurations. Moreover, the eigenvectors of the $M C E I P-\mu$ associated with higher values of $\mu$ correspond to modes having, on average, a larger number of impending slip nodes that get stuck.

For instance, for a mesh of 530 elements (41 contact candidate nodes) and for an equilibrium state having the 2 left nodes stuck, the 32 intermediate nodes in impending slip to the left and the 7 nodes on the right free, the algorithm PATH converges to a solution of the MCEIP- $\mu$ that has an unreasonably large value of $\mu$ (57.653929) and a mode represented in Fig. 4, where an impending slip node (the fourth from the left) becomes stuck. The numerical values at the contact nodes of the eigenmode represented in Fig. 4 are shown in Table 1. For the same equilibrium configuration, the solution of the classical eigenproblem corresponding to the sufficient condition (48)-(50) shows that no positive $\mu$ exists that corresponds to an admissible non-trivial solution with all impending slip nodes (s) in impending slip or in slip [the sufficient condition (48)-(50) could not be satisfied].

For the final equilibrium state of the loading process, for which the 33 nodes on the left are in impending slip and the 8 nodes on the right are free, a non-trivial eigenvector is found for a much lower coefficient of friction $(\mu=1.709967)$. The corresponding eigenmode is represented in Fig. 5. Since all the nodes in impending slip do slide, this mode satisfies also the sufficient condition (48)-(50) with $\lambda=0$. For the mesh of 776 elements, in the final steady sliding configuration, the 52 nodes on the left are in impending slip to the left and the 13 nodes on the right are free. A non-trivial eigenvector is found for a coefficient of friction $\mu=1.707375$ which is represented in Fig. 6. For the mesh of 1890 elements, in the final steady sliding configuration, the 129 nodes on the left are in impending slip to the left and the 32 nodes on the right are free. A non-trivial eigenvector is found for a coefficient of friction $\mu=1.705864$, which is represented in Fig. 7.

For other meshes and other aspect ratios $H / L$, the same trends are observed in the behavior of this system. Additional studies are needed for a better comprehension of the instability modes (Fig. 4) that cannot be detected by the sufficient condition (48)-(50) and of the circumstances at which they may arise for lower values of the friction coefficient.

\subsection{Continuation of the study of the example of Section 5.4}

In order to initiate the study of the circumstances at which all-slip and non-all-slip modes of instability may arise, we consider the block of Section 5.4 uniformly refined with rectangular bilinear finite elements with an aspect ratio height $=\frac{1}{2}$ and with $2,3,4,5,9,17$ and 33 contact nodes. In order to get solutions that may be compared with those of the single finite element in Section 5.4 (where both contact candidate nodes 
Table 1

Values at the contact nodes of the solution of the $M C E I P-\mu(\mu=57.653929)$ represented in Fig. 4

\begin{tabular}{|c|c|c|}
\hline Node & $\Xi_{s t}$ & $\Psi_{s t}$ \\
\hline 1 & 7.029992 & 0 \\
\hline 2 & 0 & 257.419724 \\
\hline 3 & 1.425031 & 0 \\
\hline 4 & 1.660202 & 0 \\
\hline 5 & 0.557874 & 0 \\
\hline 6 & 1.376425 & 0 \\
\hline 7 & 0.793989 & 0 \\
\hline 8 & 0.903294 & 0 \\
\hline 9 & 0.876847 & 0 \\
\hline 10 & 0.746456 & 0 \\
\hline 11 & 0.789053 & 0 \\
\hline 12 & 0.702387 & 0 \\
\hline 13 & 0.687086 & 0 \\
\hline 14 & 0.653370 & 0 \\
\hline 15 & 0.614355 & 0 \\
\hline 16 & 0.585934 & 0 \\
\hline 17 & 0.549948 & 0 \\
\hline 18 & 0.519051 & 0 \\
\hline 19 & 0.488578 & 0 \\
\hline 20 & 0.455087 & 0 \\
\hline 21 & 0.426626 & 0 \\
\hline 22 & 0.396458 & 0 \\
\hline 23 & 0.369891 & 0 \\
\hline 24 & 0.341483 & 0 \\
\hline 25 & 0.317338 & 0 \\
\hline 26 & 0.293616 & 0 \\
\hline 27 & 0.273702 & 0 \\
\hline 28 & 0.254264 & 0 \\
\hline 29 & 0.239737 & 0 \\
\hline 30 & 0.227570 & 0 \\
\hline 31 & 0.222595 & 0 \\
\hline 32 & 0.221762 & 0 \\
\hline
\end{tabular}

Node 1 is the third node counted from point $A$ to the right and node 32 is the 34th one. The solution is normalized such that the sum of the components of $\boldsymbol{\Xi}_{s t}$ is equal to 25 (constant $c$ in (61)).

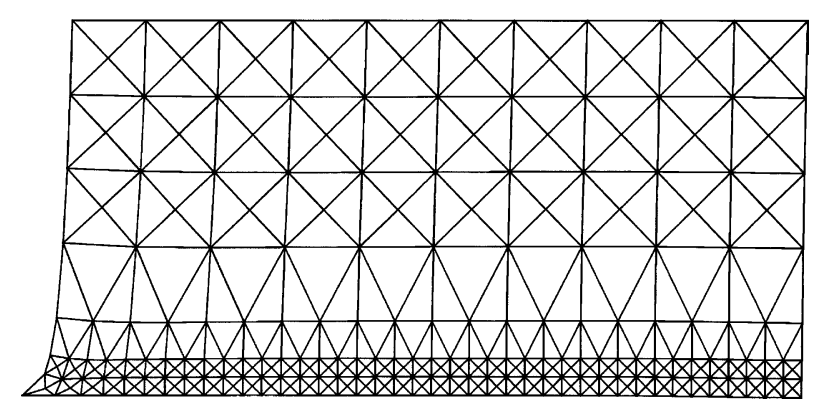

Fig. 5. An instability mode at the onset of instability for a mesh of 530 elements and $\mu=1.709967$ (solution of $M C E I P-\mu$ ).

are in impending slip to the left at the equilibrium state), we impose that all the contact candidate nodes are in a state of impending slip to the left. 


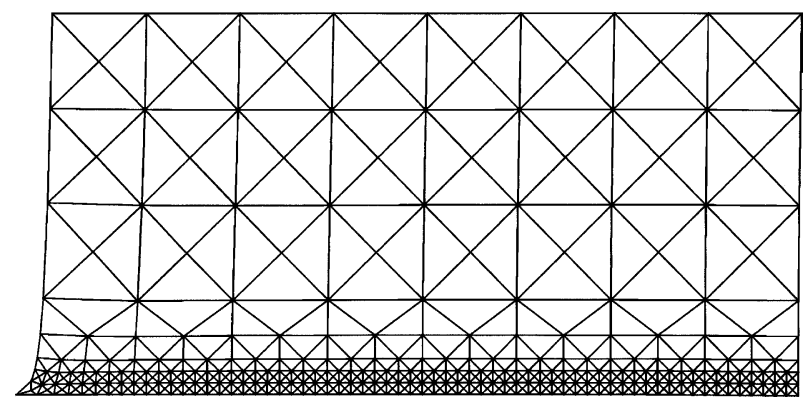

Fig. 6. An instability mode at the onset of instability for a mesh of 776 elements and $\mu=1.707375$ (solution of $M C E I P-\mu$ ).

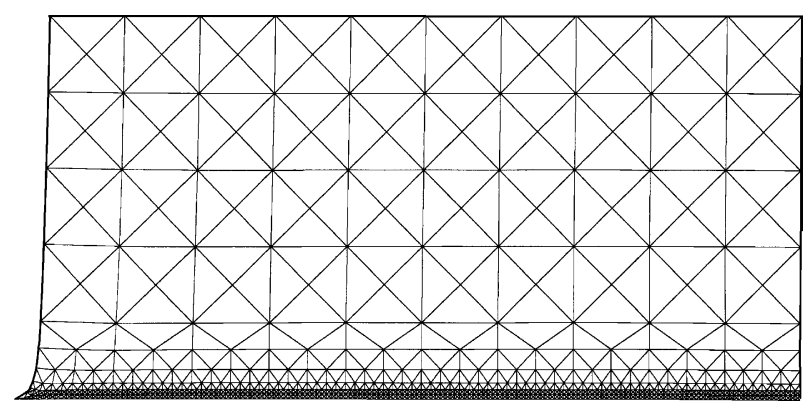

Fig. 7. An instability mode at the onset of instability for a mesh of 1890 elements and $\mu=1.705864$ (solution of $M C E I P-\mu$ ).

For a Poisson ratio of $v=0.48$, the algorithm $P A T H$ converges always to an instability mode where all the contact nodes slide to the left (an all-slip mode) (see Fig. 8). Solving all the $2^{n_{s}}$ classical generalized linear eigenproblems (34) and inequalities (36)-(40) that are equivalent to the problem $M C E I P-\mu$ for $n_{s}=2,3$ and 4 contact nodes, it is also observed that only a transition to an all-slip type of directional instability is possible in those cases.

For a Poisson ratio $v=0.1$ and for meshes with $n_{s}=2,3,4,5,9,17$ and 33 contact nodes, the algorithm $P A T H$ converges always to instability modes where all nodes slide to the left except the second node from $C$ that blocks (see Fig. 9). Solving all the $2^{n_{s}}$ classical generalized linear eigenproblems (35) and inequalities (36)-(40) that are equivalent to the problem $M C E I P-\mu$ for $n_{s}=2,3$ and 4 contact nodes, it is observed that,

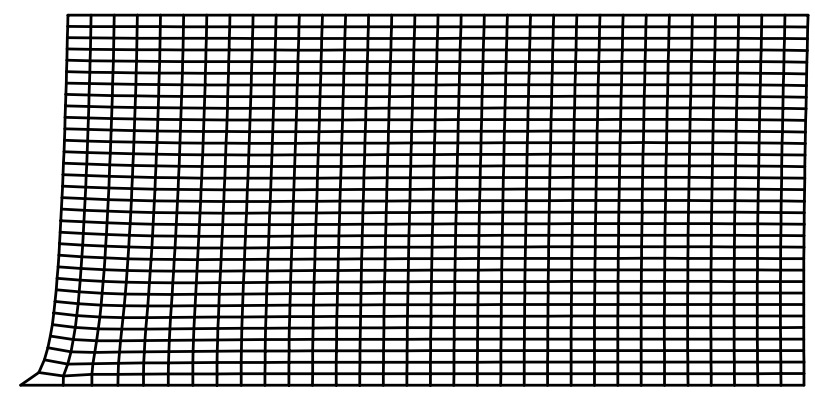

Fig. 8. An instability mode at the onset of instability (solution of $M C E I P-\mu$ ) for $\mu=1.7179983$ and $v=0.48$. All the contact nodes slide. 


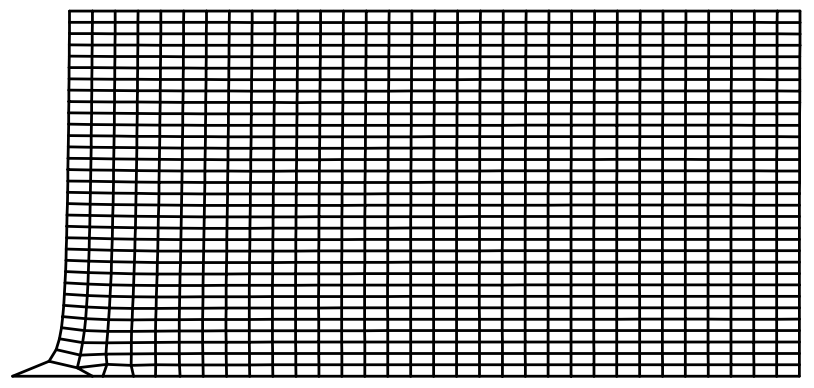

Fig. 9. Instability mode at the onset of instability (solution of $M C E I P-\mu$ ) for $\mu=3.46964934$ and $v=0.1$. All the nodes slide except the second one from the left.

Table 2

Values of $\boldsymbol{\Xi}_{s t}$ for the solutions of the $M C E I P-\mu$ at the contact nodes for different values of the Poisson ratio

\begin{tabular}{|c|c|c|c|c|}
\hline Node & $v=0.1$ & $v=0.13$ & $v=0.14$ & $v=0.48$ \\
\hline $1 \equiv A$ & 12.471171 & 12.129381 & 11.632991 & 3.410215 \\
\hline 2 & 0 & 0 & 0.242968 & 2.008466 \\
\hline 3 & 2.696330 & 2.581900 & 2.479040 & 1.611994 \\
\hline 4 & 0.954403 & 1.000953 & 1.072382 & 1.398868 \\
\hline 5 & 1.160757 & 1.138356 & 1.134683 & 1.229909 \\
\hline 6 & 0.804426 & 0.830034 & 0.857567 & 1.105762 \\
\hline 7 & 0.732580 & 0.743465 & 0.757901 & 1.007071 \\
\hline 8 & 0.609406 & 0.631130 & 0.649571 & 0.926832 \\
\hline 9 & 0.538288 & 0.558066 & 0.573943 & 0.860133 \\
\hline 10 & 0.472357 & 0.494334 & 0.510049 & 0.803677 \\
\hline 11 & 0.421753 & 0.443481 & 0.458197 & 0.755107 \\
\hline 12 & 0.378518 & 0.400407 & 0.414455 & 0.712676 \\
\hline 13 & 0.342399 & 0.363891 & 0.377200 & 0.675050 \\
\hline 14 & 0.311288 & 0.332301 & 0.344937 & 0.641202 \\
\hline 15 & 0.284324 & 0.304670 & 0.316648 & 0.610329 \\
\hline 16 & 0.260626 & 0.280210 & 0.291560 & 0.581802 \\
\hline 17 & 0.239622 & 0.258357 & 0.269099 & 0.555131 \\
\hline 18 & 0.220854 & 0.238684 & 0.248839 & 0.529930 \\
\hline 19 & 0.203991 & 0.220876 & 0.230461 & 0.505902 \\
\hline 20 & 0.188785 & 0.204702 & 0.213735 & 0.482816 \\
\hline 21 & 0.175059 & 0.189999 & 0.198495 & 0.460501 \\
\hline 22 & 0.162693 & 0.176659 & 0.184634 & 0.438835 \\
\hline 23 & 0.151613 & 0.164623 & 0.172094 & 0.417747 \\
\hline 24 & 0.141792 & 0.153877 & 0.160862 & 0.397216 \\
\hline 25 & 0.133248 & 0.144454 & 0.150975 & 0.377285 \\
\hline 26 & 0.126043 & 0.136433 & 0.142517 & 0.358077 \\
\hline 27 & 0.120284 & 0.129945 & 0.135628 & 0.339834 \\
\hline 28 & 0.116124 & 0.125170 & 0.130500 & 0.322955 \\
\hline 29 & 0.113751 & 0.122333 & 0.127372 & 0.308079 \\
\hline 30 & 0.113351 & 0.121659 & 0.126498 & 0.296186 \\
\hline 31 & 0.114998 & 0.123268 & 0.128024 & 0.288748 \\
\hline 32 & 0.118325 & 0.126812 & 0.131636 & 0.287913 \\
\hline $33 \equiv B$ & 0.120841 & 0.129573 & 0.134539 & 0.293751 \\
\hline
\end{tabular}

Node 1 is the leftmost contact node and node 33 is the rightmost contact node. The solution is normalized such that the sum of the components of $\boldsymbol{\Xi}_{s t}$ is equal to 25 (constant $c$ in (61)). 
in those cases, the transition to instability does occur with a mode of the same kind, i.e. all nodes slide to the left except the second one from $C$ that blocks, and no transition to an all-slip solution is possible.

Table 2 illustrates, for the problem $M C E I P-\mu$ with the refined mesh of Figs. 8 and 9, the transition from an all-slip mode to a non-all-slip mode that has a very localized region of left slip on the left part of the contact surface, as the Poisson ratio varies between 0.48 and 0.1 . The corresponding values of $\mu$ are the following: $\mu=1.71799829$ for $v=0.48, \mu=3.32850023$ for $v=0.14, \mu=3.42430381$ for $v=0.13$, and $\mu=3.46964934$, for $v=0.10$.

\subsection{A finite element version of the single particle example of Klarbring}

The model problem treated in this subsection consists of a finite element version of the single particle example of Klarbring [16]. The plane domain has the geometry indicated in Fig. 10. The boundary segments $C D$ and $E F$ are fixed.

The nodes in segment $A B$ may have contact with an horizontal rigid flat obstacle. This structure is assumed to be in a state of plane stress and have linear elastic behavior. The elastic properties are: modulus of elasticity $E=5 \mathrm{MPa}$ and Poisson's ratio $v=0.48$. The weight of the structure is neglected. The equilibrium configuration is the undeformed configuration represented in Fig. 10 that corresponds to a state of impending slip to the left of all the contact nodes in segment $A B$. This state of impending slip is obtained by applying on $A B$ a uniformly distributed inclined load that makes an angle with the vertical equal to the friction angle $\arctan (\mu)$.

Fig. 11 represents all-slip instability modes that solve (58) and (59) corresponding to the onset $(\lambda=0)$ of instability for three different meshes: (a) 547 linear P1 finite elements (11 contact nodes); (b) 997 (14); (c) 1997 (19). The numerical values of the components at the contact nodes of the eigenmode represented in Fig. 11(c) are shown in Table 3.

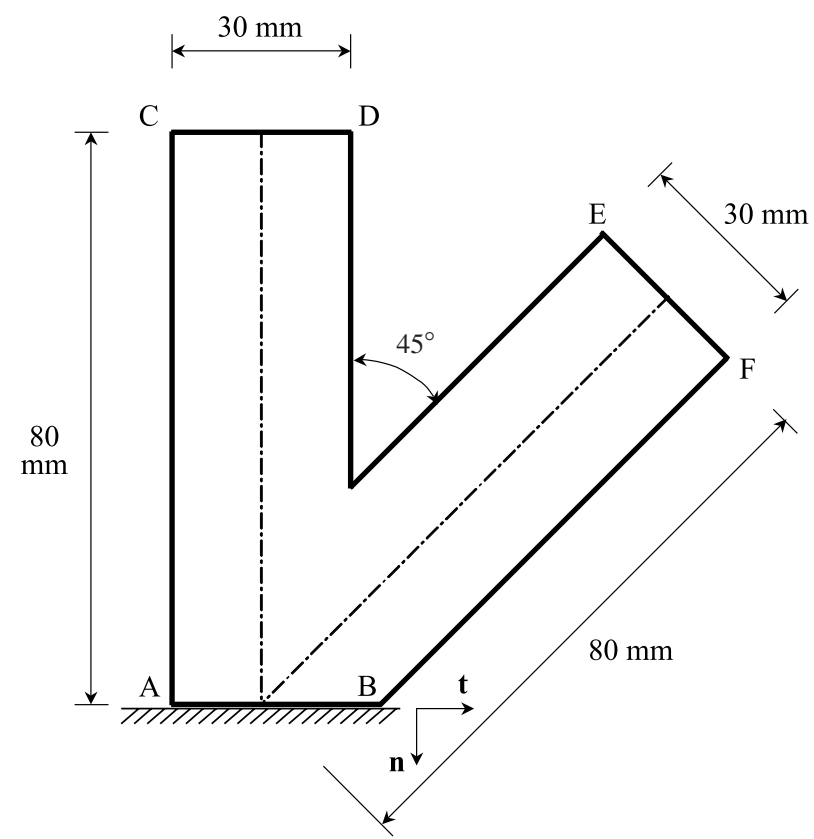

Fig. 10. Dimensions of the finite element version of the example of Klarbring [16]. 


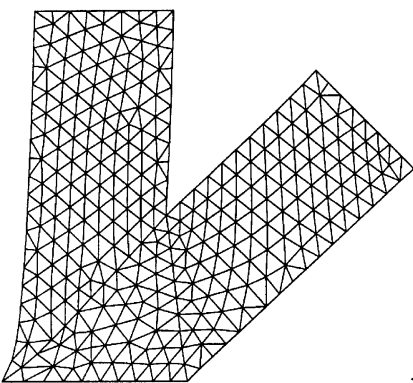

(a)

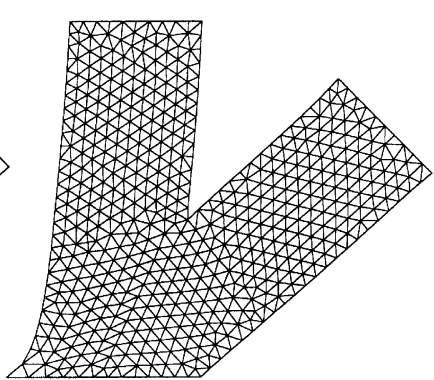

(b)

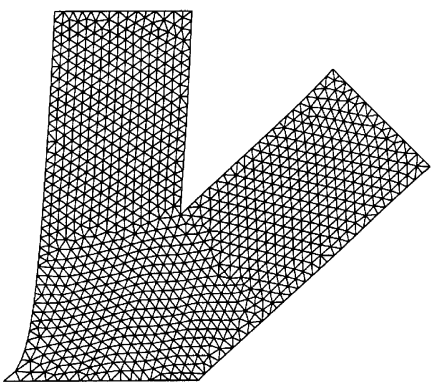

(c)

Fig. 11. Instability mode at the onset of instability for (a) $\mu=1.461772$, (b) $\mu=1.456015$ and (c) $\mu=1.452312$ (solutions of $M C E I P$ - $\mu$ ).

Table 3

Values at the contact nodes of the solution of problem MCEIP- $\mu(\mu=1.452312)$ represented in Fig. 11(c)

\begin{tabular}{lll}
\hline Node & $\boldsymbol{\Xi}_{s t}$ & $\boldsymbol{\Psi}_{s t}$ \\
\hline $1 \equiv A$ & 0.022538 & 0 \\
2 & 0.019384 & 0 \\
3 & 0.017517 & 0 \\
4 & 0.016155 & 0 \\
5 & 0.014967 & 0 \\
6 & 0.014097 & 0 \\
7 & 0.013443 & 0 \\
8 & 0.012870 & 0 \\
9 & 0.012367 & 0 \\
10 & 0.011939 & 0 \\
11 & 0.011573 & 0 \\
12 & 0.011231 & 0 \\
13 & 0.010935 & 0 \\
14 & 0.010674 & 0 \\
15 & 0.010444 & 0 \\
16 & 0.010226 & 0 \\
17 & 0.010037 & 0 \\
18 & 0.009873 & 0 \\
$19 \equiv B$ & 0.009733 & 0 \\
\hline
\end{tabular}

Equilibrium state of impending slip to the left of the 19 contact nodes. The solution is normalized such that the sum of the components of $\boldsymbol{\Xi}_{s t}$ is equal to 0.25 (constant $c$ in (61)).

The solution of the $M C E I P-\lambda^{2}$ for $\mu=5$ is discussed next. For that coefficient of friction the classical eigenproblem corresponding to the sufficient condition (48)-(50) does not have any solution satisfying the inequalities (49) and (50) (all-slip solutions). However the solutions of the non-linear eigenproblem (55) and (56) to which the PATH algorithm converges have one or more nodes that become blocked while others continue in impending slip without slip (degenerated states); as the mesh is refined the solutions successively obtained involve progressive localization of the left slip zone near the left contact node, and the corresponding positive eigenvalues $\lambda(1328.28833,3336.23641$ and 4643.97534) reveal an increased instability of those increasingly localized modes; for the meshes of Fig. 11(a)-(c) see Tables 4-6, respectively. 
Table 4

Values at the contact nodes of the solution of problem $M C E I P-\lambda^{2}(\lambda=1328.28833)$ for $\mu=5$

\begin{tabular}{lll}
\hline Node & $\boldsymbol{\Xi}_{s t}$ & $\boldsymbol{\Psi}_{s t}$ \\
\hline $1 \equiv A$ & 0.19772995 & 0 \\
2 & 0.02984346 & 0 \\
3 & 0.02048800 & 0 \\
4 & 0.00192999 & 0 \\
5 & 0 & 0.01602962 \\
6 & 0.00000845 & 0 \\
7 & 0 & 0.00000145 \\
8 & 0 & 0 \\
9 & 0 & 0 \\
10 & 0 & 0 \\
$11 \equiv B$ & 0 & 0 \\
\hline
\end{tabular}

Equilibrium state of impending slip to the left of the 11 contact nodes. The solution is normalized such that the sum of the components of $\boldsymbol{\Xi}_{s t}$ is equal to 0.25 .

Table 5

Values at the contact nodes of the solution of problem $M C E I P-\lambda^{2}(\lambda=3336.23641)$ for $\mu=5$

\begin{tabular}{lll}
\hline Node & $\boldsymbol{\Xi}_{s t}$ & $\boldsymbol{\Psi}_{s t}$ \\
\hline $1 \equiv A$ & 0.24999569 & 0 \\
2 & 0 & 0.92685590 \\
3 & 0 & 0.03493371 \\
4 & 0 & 0.00050009 \\
5 & 0 & 0.00016016 \\
6 & 0.00000403 & 0 \\
7 & 0 & 0 \\
8 & 0 & 0 \\
9 & 0 & 0.00000011 \\
10 & 0 & 0.00000109 \\
11 & 0 & 0 \\
12 & 0 & 0 \\
13 & 0 & 0 \\
$14 \equiv B$ & 0 & 0
\end{tabular}

Equilibrium state of impending slip to the left of the 14 contact nodes. The solution is normalized such that the sum of the components of $\boldsymbol{\Xi}_{s t}$ is equal to 0.25 .

\section{The relation between the directional instability and the rate problem}

\subsection{A result that takes into consideration the non-symmetry of the problem}

In this section we study the relation between (i) the problem of directional instability (divergence from an equilibrium state $\left(\mathbf{X}^{0}, \mathbf{r}^{0}\right)$ due to smooth solutions with perturbed initial conditions) and (ii) the quasi-static rate problem formulated at the same equilibrium state $\left(\mathbf{X}^{0}, \mathbf{r}^{0}\right)$. At some given instant and load, at which the equilibrium state is known, the quasi-static rate problem comprises the computation of the first order right rates of change of displacements and reactions, for a given external loading rate at the same instant $[1,16,28]$. Problem (i) involves the combined effects of mass, stiffness and friction, while problem (ii) involves the combined effects of stiffness and friction only. It is then natural to relate the above two problems on the basis of the combined effects of stiffness and friction. An angular bifurcation may occur at an equilibrium state that is not directionally unstable. This phenomenon is observed for example in the 
Table 6

Values at the contact nodes of the solution of problem $M C E I P-\lambda^{2}(\lambda=4643.97534)$ for $\mu=5$

\begin{tabular}{lll}
\hline Node & $\mathbf{\Xi}_{s t}$ & $\boldsymbol{\Psi}_{s t}$ \\
\hline $1 \equiv A$ & 0.24985631 & 0 \\
2 & 0 & 0.48831049 \\
3 & 0 & 0.01143208 \\
4 & 0.00013863 & 0 \\
5 & 0.00000436 & 0 \\
6 & 0 & 0 \\
7 & 0 & 0.00000388 \\
8 & 0 & 0 \\
9 & 0 & 0 \\
10 & 0 & 0 \\
11 & 0 & 0 \\
12 & 0 & 0 \\
13 & 0 & 0 \\
14 & 0 & 0 \\
15 & 0 & 0 \\
16 & 0 & 0 \\
17 & 0 & 0 \\
18 & 0 & 0 \\
$19 \equiv B$ & 0 & 0 \\
\hline
\end{tabular}

Equilibrium state of impending slip to the left of the 19 contact nodes. The solution is normalized such that the sum of the components of $\boldsymbol{\Xi}_{s t}$ is equal to 0.25 .

classical (associated) Shanley column [37,38] and in a non-associated (Coulomb friction) version of Shanley column [20]. In the field of associated plasticity Hill [12] and Petryk [29] and in the field of non-associated plasticity Raniecki and Bruhns [33] and Bigoni [2] discussed earlier the relation between multiplicity of solution of the quasi-static rate problem and directional instability. Nguyen [27] and Chateau and Nguyen [5] studied the same relation in the broader domain of the materials with non-associated constitutive laws and the dry friction problems. As pointed out very clearly by Bigoni [2, p. 15] the essential limitation of previous results is that they do not state that absence of bifurcation $\Rightarrow$ absence of directional instability, but they state that a sufficient condition for absence of bifurcation $\Rightarrow$ absence of directional instability. In this section we improve on these results by using properties of non-symmetric matrices that are sensitive to that non-symmetry.

We begin by reformulating the quasi-static first order rate problem with the purpose of comparing the properties of its stiffness matrix with the properties of the stiffness matrix of the problem of directional instability. After the transformation of variables (2) the rate problem is

Find $(\tilde{\mathbf{V}}, \widetilde{\mathbf{W}}) \in \mathbb{R}^{N} \times \mathbb{R}^{N}$, such that

$$
\begin{aligned}
& \widetilde{\mathbf{K}} \widetilde{\mathbf{V}}=\dot{\Lambda} \widetilde{\mathbf{L}}+\widetilde{\mathbf{W}} \\
& \widetilde{\mathbf{V}}=\left\{\begin{array}{c}
\widetilde{\mathbf{V}}_{F} \\
\mathbf{v}
\end{array}\right\}, \quad \mathbf{v} \in \mathscr{K}_{\mathbf{v}}\left(\mathbf{X}^{0}, \mathbf{r}^{0}\right), \quad \widetilde{\mathbf{W}}=\left\{\begin{array}{c}
\mathbf{0} \\
\mathbf{w}
\end{array}\right\}, \quad \mathbf{w} \in \mathscr{K}_{\mathbf{w}}\left(\mathbf{X}^{0}, \mathbf{r}^{0}\right), \\
& \mathbf{w} \cdot g(\mathbf{v})=0,
\end{aligned}
$$

where $\widetilde{\mathbf{L}}$ is the load rate direction vector and $\dot{\Lambda}$ denotes the rate of change of the load parameter. Restricting ourselves to the case of $\mathscr{P}_{z}=\emptyset$ and performing the transformation of variables (25), the rate problem becomes a mixed linear complementarity problem: 
Find $(\boldsymbol{\Xi}, \boldsymbol{\Psi}) \in \mathbb{R}^{N^{*}} \times \mathbb{R}^{N^{*}}$, such that

$$
\begin{aligned}
& \mathbf{K}^{*} \boldsymbol{\Xi}=\dot{\Lambda} \mathbf{L}^{*}+\boldsymbol{\Psi}, \\
& \boldsymbol{\Psi}_{f}=\mathbf{0}, \quad \mathbf{0} \leqslant \boldsymbol{\Xi}_{s t} \perp \boldsymbol{\Psi}_{s t} \geqslant \mathbf{0},
\end{aligned}
$$

where $\mathbf{K}^{*}=\mathbf{K}^{*}\left(\mu_{s}\right)$ is the effective stiffness matrix in (29) and (31) for the directional instability problem and

$$
\mathbf{L}^{*}=\left\{\begin{array}{ll}
\widetilde{\mathbf{L}}_{f}^{\mathrm{T}} & \left(\mathbf{S}_{s} \widetilde{\mathbf{L}}_{s t}-\mu_{s} \widetilde{\mathbf{L}}_{s n}\right)^{\mathrm{T}}
\end{array}\right\}^{\mathrm{T}} .
$$

If the principal sub-matrix $\mathbf{K}_{f, f}^{*}=\widetilde{\mathbf{K}}_{f, f}$ is invertible, it is possible to eliminate the velocities of the free degrees of freedom from problem (67) and (68): by using the definition $\check{\mathbf{K}}$ of the Schur complement of $\mathbf{K}_{f, f}^{*}$ in $\mathbf{K}^{*}$ [7] the problem (67) and (68) is rewritten in the form

Find $\left(\boldsymbol{\Xi}_{s t}, \boldsymbol{\Psi}_{s t}\right) \in \mathbb{R}^{n_{s}} \times \mathbb{R}^{n_{s}}$, such that

$$
\begin{aligned}
& \check{\mathbf{K}}^{*} \boldsymbol{\Xi}_{s t}=\dot{\Lambda} \check{\mathbf{L}}^{*}+\boldsymbol{\Psi}_{s t}, \\
& \mathbf{0} \leqslant \boldsymbol{\Xi}_{s t} \perp \boldsymbol{\Psi}_{s t} \geqslant \mathbf{0} .
\end{aligned}
$$

In the limit situation corresponding to $\lambda=0$, and performing the same condensation on the contact degrees of freedom, the problem (26)-(28) may be formulated as an homogeneous Linear Complementarity Problem $(L C P)$ :

Find $\left(\boldsymbol{\Xi}_{s t}, \boldsymbol{\Psi}_{s t}\right) \in \mathbb{R}^{n_{s}} \times \mathbb{R}^{n_{s}}$, with $\boldsymbol{\Xi}_{s t} \neq \mathbf{0}$, such that

$$
\begin{aligned}
& \check{\mathbf{K}}^{*} \boldsymbol{\Xi}_{s t}=\boldsymbol{\Psi}_{s t}, \\
& \mathbf{0} \leqslant \boldsymbol{\Xi}_{s t} \perp \boldsymbol{\Psi}_{s t} \geqslant \mathbf{0} .
\end{aligned}
$$

The coefficient matrix $\check{\mathbf{K}}^{*}$ is the same as the one in the rate problem (70) and (71). Then, the absence of (nontrivial) solution for the directional instability eigenproblem (26)-(28) for a vanishing eigenvalue $\lambda$ occurs when the previous problem has no solution, which corresponds to the case where the homogeneous $L C P$ (72) and (73) has the trivial solution $\left(\boldsymbol{\Xi}_{s t}, \boldsymbol{\Psi}_{s t}\right)=(0,0)$ as its unique solution. By definition, an homogeneous $L C P$ has the trivial solution as its unique solution if and only if its coefficient matrix is of class $R_{0}$ [7]. A matrix is of class $R_{0}$ if and only if its corresponding homogeneous $L C P$ has only the trivial solution [7]. Since a $P$ matrix is also a $R_{0}$ matrix and $\mathbf{K}^{*} \in P$ implies $\check{\mathbf{K}}^{*} \in P$ then we may state the following proposition that relates the two kinds of problems under consideration in this section.

Proposition 3. Assume that, at the current equilibrium state, there are no particles in contact with zero reaction $\left(\mathscr{P}_{z}=\emptyset\right)$. If the first order quasi-static rate problem has a unique solution for any loading rate, then the transition of the linearized system to directional instability by vanishing of an eigenvalue $\lambda$ cannot occur.

Using the same kind of words as the ones of Bigoni [2], the above statement says that absence of bifurcation implies no transition to directional instability through the vanishing of an eigenvalue $\lambda$.

\subsection{A column with Coulomb friction (a non-associative Shanley column) revisited}

This example deals with a column that has elastic supports connected to frictional sliders, as represented in Fig. 12 (see Section 4.4 of [20]). The model consists of a rigid homogeneous bar $A B$ of length $L$ and mass $M$ rigidly connected at point $B$ to a rigid massless bar orthogonal to $A B$. Point $B$ has no horizontal displacement. The column is supported by four springs of stiffness $K$ each. The rigid bars are connected to the four vertical springs by frictionless sliders $E$ and $F$. These four springs remain vertical. The particles $C$ and 

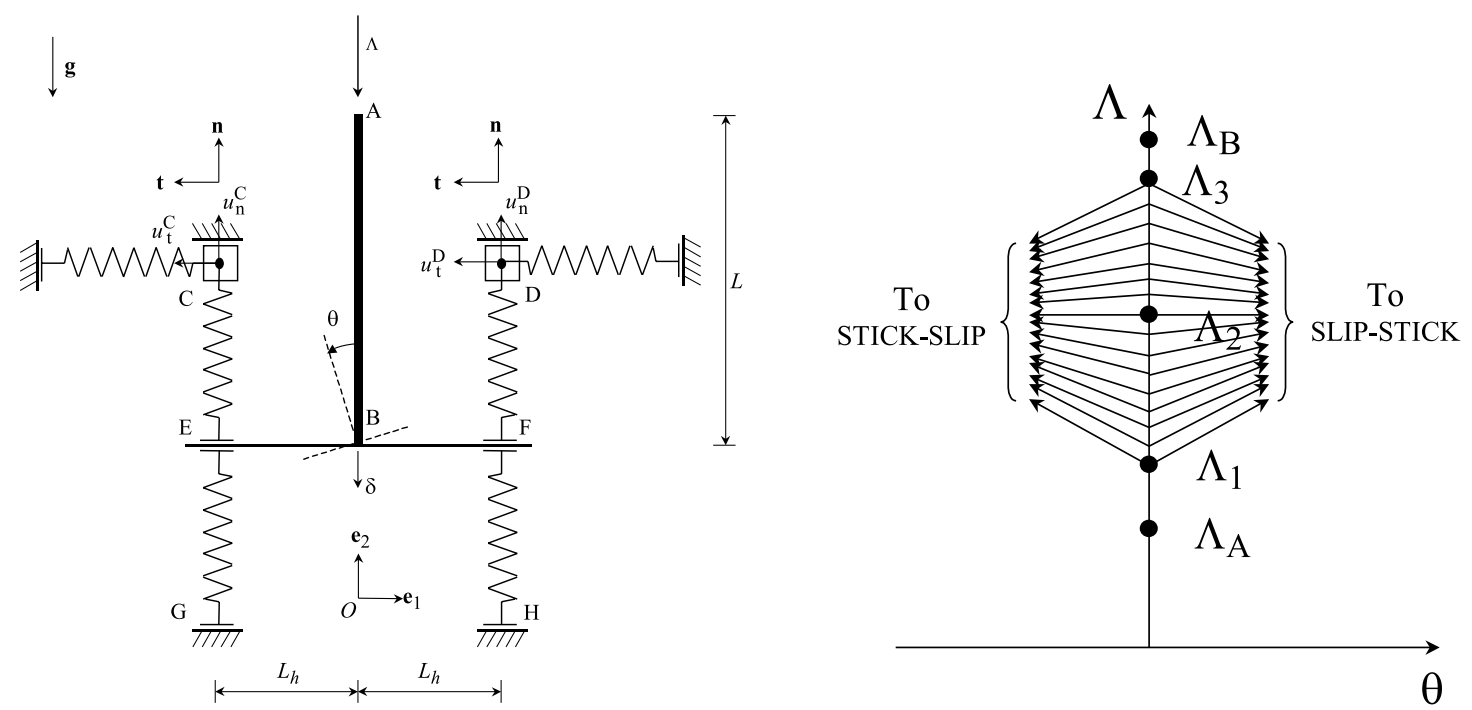

Fig. 12. On the left: a column with elastic supports attached to masses $(C$ and $D)$ in frictional contact with fixed obstacles. On the right: bifurcation diagram indicating rate solutions leading to STICK-SLIP or SLIP-STICK frictional contact states.

$D$ of mass $M^{p}$ are attached to the upper extremities of the upper vertical springs and may establish unilateral frictional contact with horizontal obstacles. The coefficient of friction is $\mu$. Particles $C$ and $D$ are also attached to horizontal springs of stiffness $K$. The initial distance of the vertical springs to the axis of symmetry is $L_{\mathrm{h}}$. A downward vertical prescribed displacement $\bar{U}_{\mathrm{v}}$ is applied to both the horizontal obstacles: $\bar{U}_{\mathrm{v}}$ is measured from the equilibrium position of the particles $C$ and $D$ when they are acted only by gravity. The extremities of the horizontal springs opposite to particles $C$ and $D$ have both an initial prescribed horizontal displacement $\bar{U}_{\mathrm{h}}$, which corresponds to an initial compression of those springs.

This mechanical system has six degrees of freedom. We choose for generalized coordinates the vertical displacement $(\delta)$ of point $B$, the angle $(\theta)$ between bar $A B$ and the horizontal $\left(u_{t}^{C}, u_{t}^{D}\right)$ and vertical $\left(u_{n}^{C}, u_{n}^{D}\right)$ components of the displacements of particles $C$ and $D$. The displacements are measured from the reference configuration that coincides with the symmetric equilibrium configuration of the system under the simultaneous action of gravity and vertical prescribed displacements $\bar{U}_{\mathrm{v}}$. The vector of non-dimensional generalized coordinates is $\mathbf{X}=\left\{\begin{array}{llllll}\delta & \theta & u_{n}^{C} & u_{n}^{D} & u_{t}^{C} & u_{t}^{D}\end{array}\right\}^{\mathrm{T}}$. The generalized coordinates with dimension of length are non-dimensionalized by dividing them by $L$. The non-dimensional prescribed displacements are $\bar{u}_{\mathrm{v}}=\bar{U}_{\mathrm{v}} / L$ and $\bar{u}_{\mathrm{h}}=\bar{U}_{\mathrm{h}} / L$. The non-dimensional external force $\Lambda$ and the non-dimensional reaction forces $\mathbf{r}=\left\{\begin{array}{llll}r_{n}^{C} & r_{n}^{D} & r_{t}^{C} & r_{t}^{D}\end{array}\right\}^{\mathrm{T}}$ are obtained from the dimensional ones by dividing them by $K L$. Time is nondimensionalized by multiplication by the factor $\sqrt{K / M}$. The fundamental trajectory of the system is represented in Fig. 12 and satisfies:

$$
\begin{aligned}
& \delta=\frac{\Lambda}{4}, \quad \theta=u_{n}^{C}=u_{n}^{D}=u_{t}^{C}=u_{t}^{D}=0 \quad \text { for } \Lambda \in\left[0, \Lambda_{A}[\right. \\
& \delta=\frac{\Lambda}{4}, \quad \theta=u_{n}^{C}=u_{n}^{D}=0, \quad u_{t}^{D}=-u_{t}^{C}=\bar{u}_{\mathrm{h}}-\frac{\mu}{2}\left(\bar{u}_{\mathrm{v}}-\frac{\Lambda}{2}\right) \quad \text { for } \Lambda \in\left[\Lambda_{A}, \Lambda_{B}[,\right. \\
& \delta=\frac{\Lambda}{2}, \quad \theta=0, \quad u_{n}^{C}=u_{n}^{D}=-\frac{\Lambda}{2}, \quad u_{t}^{D}=-u_{t}^{C}=\bar{u}_{\mathrm{h}} \quad \text { for } \Lambda \geqslant \Lambda_{B},
\end{aligned}
$$


where $\Lambda_{A}=2\left(\bar{u}_{\mathrm{v}}-\frac{2 \bar{u}_{\mathrm{h}}}{\mu}\right)$ is the load at which impending slip at $C$ and $D$ is first attained and $\Lambda_{B}=2 \bar{u}_{\mathrm{v}}$ is the load at which the reactions at $C$ and $D$ vanish. The near future frictional contact states of the two particles $C$ and $D$ is indicated by the words FREE, SLIP or STICK: for instance, a near future evolution involving slip of $C$ and stick of $D$ is indicated by SLIP-STICK.

The non-dimensional parameters that govern the behavior of the system are $m=M^{p} / M, l=L_{\mathrm{h}} / L$, $w=M g / 2 K L$ as well as $\bar{u}_{\mathrm{v}}, \bar{u}_{\mathrm{h}}$ and $\mu$ defined before. We denote by $a$ and $b$ the following non-dimensional quantities evaluated along the fundamental trajectory: $a=a\left(u_{t}^{D}, \Lambda\right)=4\left(l-u_{t}^{D}\right)^{2}-w-\Lambda, b=b\left(u_{t}^{D}, \Lambda\right)=$ $\frac{\Lambda}{2}+\mu\left(l-u_{t}^{D}\right)$. It is also convenient to define the three external loads corresponding to the following conditions that involve the quantities $a$ and $b: \Lambda=\Lambda_{1}[a=b \Lambda]$ is the load at which the SLIP-SLIP effective stiffness is singular; $\Lambda=\Lambda_{2}\left[a=\frac{b \Lambda}{2}\right]$ is the load at which the STICK-SLIP effective stiffness is singular; $\Lambda=\Lambda_{3}[a=0]$ is the load at which the STICK-STICK effective stiffness is singular. The bifurcation diagram depends qualitatively on the relative position of the two sets of loads $\Lambda_{A}, \Lambda_{B}$ and $\Lambda_{1}, \Lambda_{2}, \Lambda_{3}$. Here we only consider the case in which the governing parameters satisfy $\Lambda_{A}<\Lambda_{2}<\Lambda_{3}<\Lambda_{B}$. In this case, and for the particular type of loading considered, the only bifurcations from the fundamental path occur for $\Lambda \in\left[\Lambda_{A}, \Lambda_{B}[\right.$. The analytical study in [20] on this non-associative Shanley column concluded that, for the values of the external load $\Lambda$ corresponding to fundamental equilibrium states of impending slip of the particles $C$ and $D\left(\Lambda_{A} \leqslant \Lambda<\Lambda_{B}\right)$ there are not directional instability modes involving the sliding of both particles $C$ and $D$ (the sufficient condition (48)-(50) for directional instability is not satisfied) (see Fig. 12). This means that the linearized system is still directionally stable after the rate problem ceases to have a unique solution. However, when $\Lambda>\Lambda_{2}$ (the effective stiffness matrices corresponding to the slip of one contact particle and to the stick of the other contact particle are not positive definite), there exist two modes of instability that solve problem (26)-(28) and involve the slip of one particle and the stick of the other particle.

The statement of Proposition 3 is consistent with the results in [20]. In the case illustrated in Fig. 12, both the contact particles $C$ and $D$ belong to $\mathscr{P}_{s}\left(\mathscr{P}_{z}=\emptyset\right)$ for load parameters between $\Lambda_{A}$ and $\Lambda_{1}(a>b \Lambda)$, and the rate problem (70) and (71) can be written in the equivalent complementarity form

$$
\begin{aligned}
& {\left[\begin{array}{cccc}
1 & 0 & -\left(1-\frac{b \Lambda}{2 a}\right) & -\frac{b \Lambda}{2 a} \\
0 & 1 & -\frac{b \Lambda}{2 a} & -\left(1-\frac{b \Lambda}{2 a}\right)
\end{array}\right]\left\{\begin{array}{l}
\psi_{s t}^{C} \\
\psi_{s t}^{D} \\
\Xi_{s t}^{C} \\
\Xi_{s t}^{D}
\end{array}\right\}=\dot{\Lambda}\left\{\begin{array}{l}
-\mu / 4 \\
-\mu / 4
\end{array}\right\},} \\
& \mathbf{0} \leqslant\left\{\begin{array}{l}
\Xi_{s t}^{C} \\
\Xi_{s t}^{D}
\end{array}\right\} \perp\left\{\begin{array}{l}
\psi_{s t}^{C} \\
\psi_{s t}^{D}
\end{array}\right\} \geqslant \mathbf{0} .
\end{aligned}
$$

In the above range of equilibrium states $\left(\Lambda \in\left[\Lambda_{A}, \Lambda_{1}[)\right.\right.$ of the fundamental trajectory, the directional instability problem $M C E I P-\mu$ (72) and (73) is the homogeneous particular case $(\dot{\Lambda}=0)$ of (74) and (75). Then Proposition 3 guarantees that in that range of $\Lambda$ the transition to directional instability of the linearized system cannot occur for a vanishing eigenvalue $\lambda$, which is consistent with the results in Section 4.4 of [20].

The study of a $L C P$ with two pairs of variables can be interpreted graphically in $\mathbb{R}^{2}$. The problem (74) and (75) can be expressed as a non-negative linear combination of the four columns of the coefficient matrix, with two complementarity conditions:

$$
\begin{aligned}
& \psi_{s t}^{C} \mathbf{I}_{\cdot 1}+\psi_{s t}^{D} \mathbf{I}_{\cdot 2}+\Xi_{s t}^{C}\left(-\check{\mathbf{K}}_{\cdot 1}^{*}\right)+\Xi_{s t}^{D}\left(-\check{\mathbf{K}}_{\cdot 2}^{*}\right)=\dot{\Lambda}\left\{\begin{array}{c}
-\mu / 4 \\
-\mu / 4
\end{array}\right\}, \\
& \Xi_{s t}^{C}, \Xi_{s t}^{D}, \psi_{s t}^{C}, \psi_{s t}^{D} \geqslant 0,
\end{aligned}
$$




$$
\Xi_{s t}^{C} \psi_{s t}^{C}=\Xi_{s t}^{D} \psi_{s t}^{D}=0
$$

where $\mathbf{I}_{1}$ and $\check{\mathbf{K}}_{\cdot 1}^{*}$ denote column vectors containing the first columns of matrices $\mathbf{I}$ and $\check{\mathbf{K}}^{*}$, respectively, and $\mathbf{I}_{.2}$ and $\check{\mathbf{K}}_{\cdot 2}^{*}$ denote column vectors containing the second columns of matrices $\mathbf{I}$ and $\check{\mathbf{K}}^{*}$, respectively,

$$
\mathbf{I}_{\cdot 1}=\left\{\begin{array}{l}
1 \\
0
\end{array}\right\}, \quad \mathbf{I}_{\cdot 2}=\left\{\begin{array}{l}
0 \\
1
\end{array}\right\}, \quad \check{\mathbf{K}}_{\cdot 1}^{*}=\left\{\begin{array}{c}
1-\frac{b \Lambda}{2 a} \\
\frac{b \Lambda}{2 a}
\end{array}\right\}, \quad \check{\mathbf{K}}_{\cdot 2}^{*}=\left\{\begin{array}{c}
\frac{b \Lambda}{2 a} \\
1-\frac{b \Lambda}{2 a}
\end{array}\right\} .
$$

In each pair of variables $\left(\Xi_{s t}^{C}, \psi_{s t}^{C}\right)$ and $\left(\Xi_{s t}^{D}, \psi_{s t}^{D}\right)$, only one variable may be non-zero, due to the complementarity conditions (78). Consequently, the left-hand side of (76) is the non-negative linear combination of only two column vectors: $\mathbf{I}_{1}$ with $\mathbf{I}_{2}$ or $-\check{\mathbf{K}}_{1}^{*}$ with $-\check{\mathbf{K}}_{\cdot 2}^{*}$ or $\mathbf{I}_{1}$ with $-\check{\mathbf{K}}_{\cdot 2}^{*}$ or $-\check{\mathbf{K}}_{1}^{*}$ with $\mathbf{I}_{2}$. Each of these four possible non-negative combinations correspond to a complementarity cone [25]

$$
\operatorname{Pos}\left(\mathbf{I}_{1}, \mathbf{I}_{2}\right), \quad \operatorname{Pos}\left(-\check{\mathbf{K}}_{\cdot 1}^{*},-\check{\mathbf{K}}_{\cdot 2}^{*}\right), \quad \operatorname{Pos}\left(\mathbf{I}_{\cdot 1},-\check{\mathbf{K}}_{\cdot 2}^{*}\right), \quad \operatorname{Pos}\left(-\check{\mathbf{K}}_{\cdot 1}^{*}, \mathbf{I}_{\cdot 2}\right)
$$
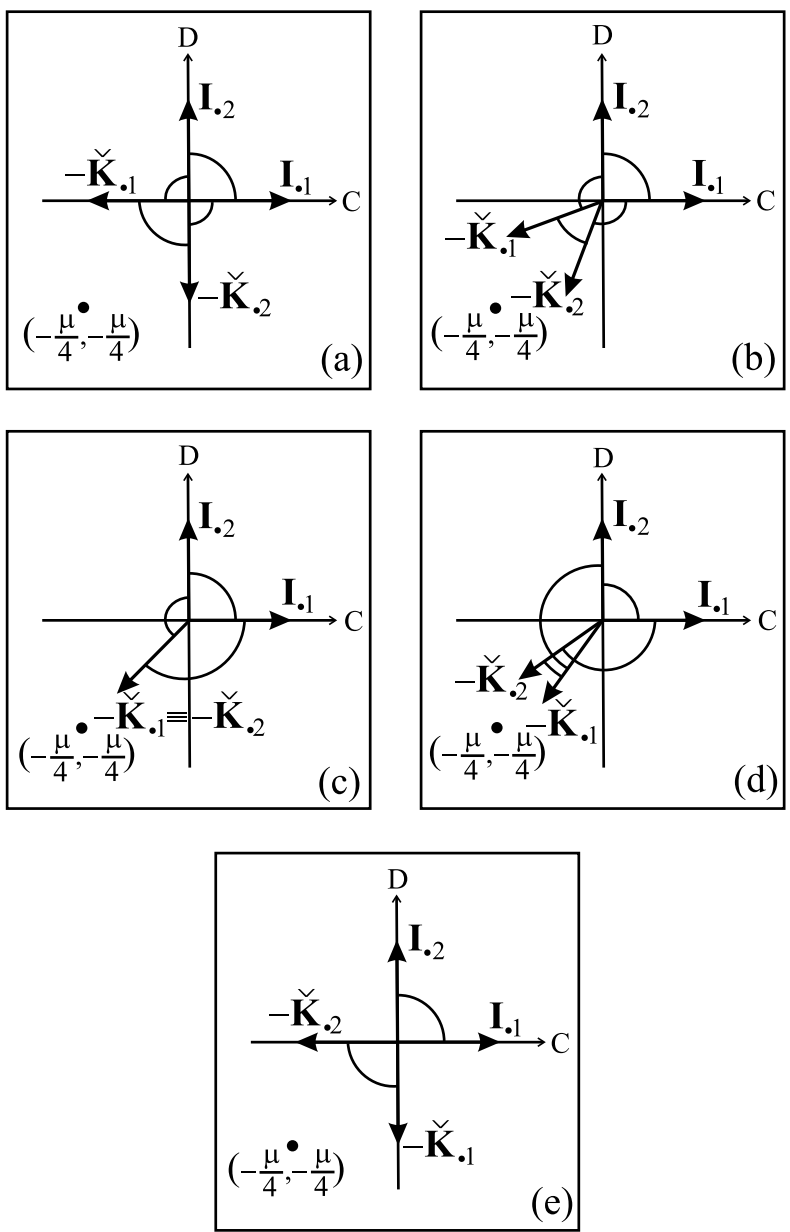

Fig. 13. Complementary cones of the rate problem (74) and (75) for the system schematically represented in Fig. 12: (a) $\Lambda=0$; (b) $0<\Lambda<\Lambda_{1}(a>b \Lambda>0)$; (c) $\Lambda=\Lambda_{1}(a=b \Lambda)$; (d) $\Lambda_{1}<\Lambda<\Lambda_{2}\left(b \frac{\Lambda}{2}<a<b \Lambda\right)$; (e) $\Lambda=\Lambda_{2}\left(a=b \frac{\Lambda}{2}\right)$. 
where $\operatorname{Pos}\left(\mathbf{a}_{1}, \mathbf{a}_{2}\right)=\left\{\mathbf{x} \in \mathbb{R}^{2}: \mathbf{x}=\alpha_{1} \mathbf{a}_{1}+\alpha_{2} \mathbf{a}_{2}, \alpha_{1}, \alpha_{2} \geqslant 0\right\}$ is the cone associated with the extreme vectors $\mathbf{a}_{1}$ and $\mathbf{a}_{2}$. The $L C P$ (74) and (75) corresponding to the rate problem is equivalent to the problem of finding the complementarity cone(s) that contains(n) the point $\dot{\Lambda}\left(-\frac{\mu}{4},-\frac{\mu}{4}\right)[25]$. The homogeneous $L C P(\dot{\Lambda}=0)$ corresponding to the onset $(\lambda=0)$ of directional instability $(M C E I P-\mu)$ is equivalent to the problem of finding the non-negative linear combinations of the column vectors $\mathbf{I}_{1}, \mathbf{I}_{2},-\check{\mathbf{K}}_{\cdot 1}^{*},-\check{\mathbf{K}}_{2}^{*}$ that are admissible by the complementarity conditions and equal to the null vector. Non-trivial solutions of an homogeneous $L C P$ can only occur if and only if its coefficient matrix is not a $R_{0}$ matrix, that is, if at least one of the complementary cones is strongly degenerated [25]. A cone $\operatorname{Pos}\left(\mathbf{a}_{1}, \mathbf{a}_{2}\right)$ is strongly degenerate if and only if its extreme vectors $\mathbf{a}_{1}$ and $\mathbf{a}_{2}$ have the same direction and opposite senses.

Fig. 13 illustrates the complementary cones of the problem (74) and (75) for several values of the loading parameter $\Lambda$. Each complementary cone $\operatorname{Pos}\left(\mathbf{a}_{1}, \mathbf{a}_{2}\right)$ is indicated by an arc of circumference connecting its two extreme vectors $\mathbf{a}_{1}$ and $\mathbf{a}_{2}$. For $0 \leqslant \Lambda<\Lambda_{1}$, i.e., for $a>b \Lambda \geqslant 0$ (Fig. 13(a) and (b)) the matrix $\check{\mathbf{K}}^{*}$ is of class $P$ and of class $R_{0}$ which means that both the non-homogeneous and the homogeneous $L C P(74)$ and (75) corresponding to the rate problem and to the $M C E I P-\mu$ have unique solutions; in this case the complementary cones are a partition of $\mathbb{R}^{2}$ and none of them is degenerate. For $\Lambda=\Lambda_{1}$, i.e., for $a=b \Lambda$ (Fig. 13(c)), $\operatorname{Pos}\left(-\check{\mathbf{K}}_{\cdot 1}^{*},-\check{\mathbf{K}}_{2}^{*}\right)$ is weakly degenerate. A cone $\operatorname{Pos}\left(\mathbf{a}_{1}, \mathbf{a}_{2}\right)$ is weakly degenerate if it is an halfline. For that load the matrix $\check{\mathbf{K}}^{*}$ is degenerate, hence it is not of class $P$ but it is of class $P_{0}$ (all its principal minors are non-negative) and of class $R_{0}$. Assuming a unit positive load rate $\dot{\Lambda}=+1$ the vector of independent terms $\left(-\frac{\mu}{4},-\frac{\mu}{4}\right)$ is on the degenerate cone $\operatorname{Pos}\left(-\check{\mathbf{K}}_{.1}^{*},-\check{\mathbf{K}}_{\cdot 2}^{*}\right)$ which corresponds to the existence of an infinity (fan) of first order rate solutions for $\Lambda=\Lambda_{1}$ (see Fig. 9 in Section 4.4 of [20]). Since the matrix $\check{\mathbf{K}}^{*}$ is of class $R_{0}$, the linearized system is still directionally stable. For $\Lambda_{1}<\Lambda<\Lambda_{2}$, i.e., for $b \frac{\Lambda}{2}<a<b \Lambda$ (Fig. 13(d)) the matrix $\check{\mathbf{K}}^{*}$ is not of class $P$. For the assumed positive load rate the quasi-static rate problem has three solutions: STICK-SLIP (associated with $\operatorname{Pos}\left(\mathbf{I}_{1},-\check{\mathbf{K}}_{\cdot 2}^{*}\right.$ )), SLIP-STICK (associated with $\left.\operatorname{Pos}\left(-\check{\mathbf{K}}_{1}^{*}, \mathbf{I}_{2}\right)\right)$ and the symmetric SLIP-SLIP evolution along the fundamental trajectory (associated with $\operatorname{Pos}\left(-\check{\mathbf{K}}_{1}^{*},-\check{\mathbf{K}}_{\cdot 2}^{*}\right)$ ). For the same load range the matrix is $\check{\mathbf{K}}^{*}$ is of class $R_{0}$ which guarantees the directional stability of the linearized system. For $\Lambda=\Lambda_{2}$, i.e., for $a=b \frac{\Lambda}{2}$ (Fig. 13(e)) the matrix $\check{\mathbf{K}}^{*}$ is neither of class $P$ nor of class $R_{0}$; the complementary cones $\operatorname{Pos}\left(\mathbf{I}_{1},-\check{\mathbf{K}}_{\cdot 2}^{*}\right)$ and $\operatorname{Pos}\left(-\check{\mathbf{K}}_{1}^{*}, \mathbf{I}_{2}\right)$ are strongly degenerate. That point of the fundamental trajectory corresponds to the onset $(\lambda=0)$ of directional instability.

\section{Conclusions}

In this paper we have presented a study on the directional instability of plane finite dimensional systems in unilateral frictional contact with curved obstacles, and on its relation with the first order quasi-static rate problem. The study of the directional instability involves the solution of a mixed explicit complementarity eigenproblem (19) and (20) which is equivalent to a mixed complementarity-inclusion eigenproblem (26)(28), and also to $a$ set of classical generalized linear eigenproblems (34) plus some inequalities (36)-(40). For computational purposes, those non-smooth eigenproblems are transformed into mixed complementarity problems (MCPS) that are solved with the $P A T H$ Mathematical Programming algorithm.

The sufficient condition for absence of directional instability given in Proposition 2 is that the nonsymmetric interval matrix $\mathbb{\square}_{z}\left(\mu_{z}\right)\left(\lambda^{2} \mathbf{M}^{*}\left(\mu_{s}\right)+\mathbf{K}^{*}\left(\mu_{s}\right)\right)$ (45) has the property $P$ for all $\lambda \geqslant 0$; in the linear elasticity case this holds if the friction coefficients $\mu_{s}$ and $\mu_{z}$ are sufficiently small and the obstacle curvature $\chi$ is sufficiently small when positive. When, at the equilibrium state, there is no particle in contact with zero reaction, the absence of directional instability holds when the pencil of effective mass and stiffness matrices $\lambda^{2} \mathbf{M}^{*}\left(\mu_{s}\right)+\mathbf{K}^{*}\left(\mu_{s}\right)$ is $P$ for all $\lambda \geqslant 0$. The $P$ property captures the non-associated (non-symmetric) character of the problem and the above conditions give values for the coefficient of friction at which directional instability initiates that are less conservative (that are higher) than those given by other conditions (47) that involve the positive definiteness property. 
In the finite element simulations with the block of Progri, Villechaise and Zeghloul [32,40], it has been observed that: (i) whenever solutions were known to exist [the all-slip solutions of the sufficient condition (48)-(50)] the PATH algorithm always converged to one such all-slip solution; (ii) in some cases where allslip solutions did not exist [the sufficient condition (48)-(50) could not be satisfied] other solutions with both slip and stick were obtained, but only for very large values of $\mu$; (iii) for reasonably small values of $\mu$, no solutions different from the all-slip solutions provided by the sufficient condition (48)-(50) were found; (iv) in all cases, the values obtained for the coefficient of friction at which directional instability could occur were larger than the experimentally observed ones.

In all the studied numerical examples, the convergence of the PATH algorithm to an all-slip solution (48)-(50) has been always observed whenever such kind of solution exists. When that is not the case, it has been frequently observed that the converged results given by that algorithm had some very localized regions of slip on the contact surface, which might localize even further as the mesh is refined. The geometry of the body and the Poisson ratio play an important role on the type of directional instability mode (all-slip or non-all-slip) that may occur for sufficiently large values of the coefficient of friction.

For a given equilibrium state of a system in which there are no particles in contact with zero reaction, we show that the uniqueness of solution of the rate problem for every loading rate direction excludes the onset $(\lambda=0)$ of directional instability (Proposition 3 ). The relation between the directional instability problem and the rate problem was illustrated with a six degree of freedom system that is a non-associated version of Shanley's column. The verification or not of the properties $P, P_{0}$ and $R_{0}$ by an effective stiffness matrix, along the fundamental equilibrium path of that system, has been related to the loss of uniqueness and the onset of directional instability along that path.

\section{References}

[1] L.-E. Andersson, A. Klarbring, Existence and uniqueness for quasistatic contact problems with friction, in: J.A.C. Martins, M.D.P. Monteiro Marques (Eds.), Contact Mechanics, Proceedings of the 3rd Contact Mechanics International Symposium, Praia da Consolação, Peniche, Portugal, 17-21 June 2001, Solid Mechanics and Its Applications, vol. 103, Kluwer Academic Publishers, 2002, pp. 245-260.

[2] D. Bigoni, Bifurcation and instability of non-associative elastoplastic solids, in: H. Petryk (Ed.), Material Instabilities in Elastic and Plastic Solids, CISM Courses and Lectures, vol. 414, Springer-Verlag, 2000, pp. 1-52.

[3] G. Björkman, Path following and critical points for contact problems, Comput. Mech. 10 (1992) 231-246.

[4] W.F. Brace, J.D. Byerlee, Stick slip as a mechanism for earthquakes, Science 153 (1966) 990-992.

[5] X. Chateau, Q.-S. Nguyen, Buckling of elastic structures in unilateral contact with or without friction, Eur. J. Mech., A/Solids 10 (1) (1991) 71-89.

[6] J.A.C. Martins, M. Raous (Eds.), Friction and Instability, CISM Courses and Lecture, vol. 457, Springer-Verlag Wien, New York, 2002.

[7] R.W. Cottle, J.-S. Pang, R.E. Stone, The Linear Complementarity Problem, Academic Press, Boston, 1992.

[8] S.P. Dirkse, M.C. Ferris, The PATH solver: a non-monotone stabilization scheme for mixed complementarity problems, Optim. Methods Software 5 (1995) 123-156.

[9] J. Duran, Sands, Powders and Grains: An Introduction to the Physics of Granular Materials, Springer-Verlag, 2000.

[10] S.W.E. Earles, C.K. Lee, Instabilities arising from the frictional interaction of a pin-disk system resulting in noise generation, ASME, J. Engrg. Ind. (February) (1976) 81-86.

[11] S.W.E. Earles, M.N.M. Badi, Oscillatory instabilities generated in a double-pin and disc undamped system: a mechanism of discbrake squeal, Proc. Inst. Mech. Engrs. 198C (4) (1984) 43-50.

[12] R. Hill, A general theory of uniqueness and stability in elastic-plastic solids, J. Mech. Phys. Solids 6 (1958) $236-249$.

[13] R.A. Ibrahim, Friction-induced vibration, chatter, squeal and chaos. Part I: Mechanics of contact and friction, Appl. Mech. Rev. 47 (7) (1994) 209-226.

[14] R.A. Ibrahim, Friction-induced vibration, chatter, squeal and chaos. Part II: Dynamics and modeling, Appl. Mech. Rev. 47 (7) (1994) 227-253.

[15] A. Klarbring, On discrete and discretized non-linear elastic structures in unilateral contact (stability, uniqueness and variational principles), Int. J. Solids Struct. 24 (5) (1988) 459-479. 
[16] A. Klarbring, Examples of non-uniqueness and non-existence of solutions to quasistatic contact problems with friction, IngenieurArchiv. 56 (1990) 529-541.

[17] A. Klarbring, G. Björkman, Solution of large displacement contact problems with friction using Newton's method for generalized equations, Int. J. Num. Meth. Engrg. 34 (1992) 249-269.

[18] A. Klarbring, Stability and critical points in large displacement frictionless contact problems, in: J.A.C. Martins, M. Raous (Eds.), Friction and Instabilities, CISM Courses and Lectures, vol. 457, Springer-Verlag, 2002, pp. 39-64.

[19] J.A.C. Martins, S. Barbarin, M. Raous, A. Pinto da Costa, Dynamic stability of finite dimensional linearly elastic systems with unilateral contact and Coulomb friction, Comput. Methods Appl. Mech. Engrg. 177 (1999) 289-328.

[20] J.A.C. Martins, A. Pinto da Costa, Stability of finite-dimensional nonlinear elastic systems with unilateral contact and friction, Int. J. Solids Struct. 37 (18) (2000) 2519-2564.

[21] J.A.C. Martins, A. Pinto da Costa, F.M.F. Simões, Some notes on friction and instabilities, in: J.A.C. Martins, M. Raous (Eds.), Friction and Instabilities, CISM Courses and Lectures, vol. 457, Springer-Verlag, Wien, New York, 2002, pp. 65-136.

[22] R.E. Moore, Methods and Applications of Interval Analysis, SIAM — Studies in Applied Mathematics, 1979.

[23] Z. Mróz, R.H. Plaut, On the stability and post-critical behavior of elastic structures with dry friction, Int. J. Solids Struct. 29 (10) (1992) 1241-1253.

[24] Z. Mróz, Contact friction models and stability problems, in: J.A.C. Martins, M. Raous (Eds.), Friction and Instabilities, CISM Courses and Lectures, vol. 457, Springer-Verlag, 2002, pp. 179-232.

[25] K.G. Murty, Linear Complementarity, Linear and Nonlinear Programming, 1997. Available from <http: //www-pers onal. engin. umich. edu/ murty>.

[26] A. Neumaier, Interval methods for systems of equations, in: G.-C. Rota (Ed.), Encyclopedia of Mathematics and its Applications, Cambridge University Press, 1990.

[27] Q.-S. Nguyen, Stabilité et bifurcation des systèmes dissipatifs standards à comportement indépendant du temps physique, C.R. Acad. Sci. Paris 310 (Série II) (1990) 1375-1380.

[28] Q.-S. Nguyen, Stabilité et Mécanique Non-Linéaire, Hermes Science Publications, Paris, 2000.

[29] H. Petryk, Theory of bifurcation and instability in time-independent plasticity, in: Q.S. Nguyen (Ed.), Bifurcation and Stability of Dissipative Systems, CISM Courses and Lectures, vol. 327, Springer, Wien, New York, 1993, pp. 95-152.

[30] H. Petryk, Material instabilities in elastic and plastic solids, in: CISM Courses and Lectures, vol. 414, Springer, Wien, New York, 2000.

[31] A. Pinto da Costa, I.N. Figueiredo, J.J. Júdice, J.A.C. Martins, A complementarity eigenproblem in the stability analysis of finite dimensional elastic systems with frictional contact, in: M.C. Ferris, O.L. Mangasarian, J.-S. Pang (Eds.), Complementarity: Applications, Algorithms and Extensions, Kluwer Academic Publishers, Dordrecht, 2001, p. 50, 67-83.

[32] R. Progri, B. Villechaise, Analyse des glissements dans un contact sec, C.R. Acad. Sci. Paris 299 (Série II, 12) (1984) $763-768$.

[33] B. Raniecki, O.T. Bruhns, Bounds to bifurcation stresses in solids with non-associated plastic flow law at finite strain, J. Mech. Phys. Solids 29 (2) (1981) 153-172.

[34] J.R. Rice, A.L. Ruina, Stability of steady frictional slipping, ASME, J. Appl. Mech. 50 (1983) $343-349$.

[35] J.R. Rice, New perspectives on crack and fault dynamics, in: H. Aref, J.W. Philips (Eds.), Mechanics for a New Millenium, Kluwer Academic Publishers, 2001, pp. 1-24.

[36] C.H. Scholz, The Mechanics of Earthquakes and Faulting, Cambridge University Press, Cambridge, UK, 1990.

[37] F.R. Shanley, The column paradox, J. Aeronaut. Sci. 13 (12) (1946) 678.

[38] F.R. Shanley, Inelastic column theory, J. Aeronaut. Sci. 14 (5) (1947) 261-267.

[39] D. Vola, M. Raous, J.A.C. Martins, Friction and instability of steady sliding: squeal of a rubber/glass contact, Int. J. Num. Meth. Engrg. 46 (10) (1999) 1699-1720.

[40] T. Zeghloul, B. Villechaise, Stress waves in a sliding contact, Part 1: Experimental study, in: Proc. 22nd Leeds-Lyon symposium on Tribology, 5-8 September 1995. 